\title{
A Comprehensive Assessment of Dialog Evaluation Metrics
}

\author{
Yi-Ting Yeh, Maxine Eskenazi, Shikib Mehri \\ Language Technologies Institute, Carnegie Mellon University \\ \{yitingye, max, amehri\} @cs.cmu.edu
}

\begin{abstract}
Automatic evaluation metrics are a crucial component of dialog systems research. Standard language evaluation metrics are known to be ineffective for evaluating dialog. As such, recent research has proposed a number of novel, dialog-specific metrics that correlate better with human judgements. Due to the fast pace of research, many of these metrics have been assessed on different datasets and there has as yet been no time for a systematic comparison between them. To this end, this paper provides a comprehensive assessment of recently proposed dialog evaluation metrics on a number of datasets. In this paper, 23 different automatic evaluation metrics are evaluated on 10 different datasets. Furthermore, the metrics are assessed in different settings, to better qualify their respective strengths and weaknesses. This comprehensive assessment offers several takeaways pertaining to dialog evaluation metrics in general. It also suggests how to best assess evaluation metrics and indicates promising directions for future work.
\end{abstract}

\section{Introduction}

Evaluation is a crucial component of the research process. Evaluation metrics are used to shine light on the best models and thus they strongly influence the research directions of a field. Standard automatic language evaluation metrics (e.g., BLEU, METEOR) have been shown to be ineffective for evaluating dialog (Liu et al., 2016b; Deriu et al., 2021). To this end, recent research has proposed a number of automatic metrics specifically designed to evaluate dialog (Tao et al., 2018; Ghazarian et al., 2019; Lan et al., 2020; Pang et al., 2020; Ghazarian et al., 2020; Sinha et al., 2020; Huang et al., 2020; Mehri and Eskenazi, 2020b,a; Zhang et al., 2021b). These metrics address the weaknesses of the standard language evaluation metrics. They have also been shown to better correlate with human judgement. However, most of these metrics were devel- oped at about the same time and were evaluated on different datasets. As such, there has not yet been a consistent comparison amongst them. This paper describes an assessment of these metrics on several different datasets. It quantifies the relative performance and reveals strengths and weaknesses of each metric.

Due to the fact that standard automatic metrics have been shown to be ineffective for dialog evaluation (Liu et al., 2016b; Deriu et al., 2021), dia$\log$ research typically relies on human evaluation. While useful and very informative, human evaluation is expensive and time-consuming. Given these considerations, at present it is usually only used for a final evaluation. Yet, during the development process, automatic evaluation metrics are essential since they are used to optimize the model design and the choice of hyperparameters. In order to be relevant and meaningful, these metrics must better correlate with human judgment so that they serve as a meaningful proxy for human judgements during the development process.

This paper discusses the assessment of several recently-proposed automatic evaluation metrics for dialog over several datasets. These datasets have human annotations that measure the quality of the responses. Furthermore, this paper includes analysis experiments that qualify the performance of the metrics across different settings. This assessment measures the performance of the metrics (1) on both the turn level and the dialog level, (2) for different dialog lengths, (3) for different dialog qualities (e.g., coherence, engaging), (4) for different types of response generation models (i.e., generative, retrieval, simple models and state-ofthe-art models), and (5) exploring combinations of different metrics.

All of the code created for this assessment is open-sourced ${ }^{1}$ to facilitate the easy assessment of

\footnotetext{
1https://github.com/exe1023/ DialEvalMetrics
} 
future evaluation metrics on a number of datasets.

\section{Overview of Automatic Metrics}

In this paper, we studied 23 automatic metrics either designed for evaluating dialogues or general natural language generation (NLG) tasks. Table 1 presents an overview of metrics. Besides model designs and training objectives, the three aspects used to characterize them are: (1) Does the metric use a pretrained language model? (2) What data was used to train the metric? (3) Does the metric require a reference response or is it reference-free?

In most metrics, triplet ranking loss is used as a training objective, and it requires the model to give higher scores to appropriate responses. BERT (Devlin et al., 2019) and its variant RoBERTa (Liu et al., 2019) are the most popular pretrained language models utilized in state-of-the-art metrics, followed by GPT-2 language model (Radford et al., 2019). DailyDialog (Li et al., 2017) and PersonaChat (Zhang et al., 2018) are two widely-used datasets for training metrics. Briefly, conversation topics of DailyDialog are about day-to-day life, while Personachat consists of dialogs where each participant is assigned a persona and the goal is to become familiar with the other individual. Wordoverlap metrics are ineffective for dialog (Liu et al., 2016b) largely due to the one-to-many nature of dialog (Zhao et al., 2017a). Thus, reference-free metrics have been proposed to circumvent the oneto-many problem. Amongst the 24 metrics assessed here, we have 11 reference-free evaluation metrics.

We refer readers to Appendix A for detailed discussion of aforementioned characteristics of assessed metrics.

\section{Testing Datasets}

In this work, we test metrics with the quality annotated datasets: USR (Mehri and Eskenazi, 2020b), GRADE (Huang et al., 2020), HolisticEval (Pang et al., 2020), FED (Mehri and Eskenazi, 2020a), DSTC6 (Hori and Hori, 2017) and DSTC9 (Gunasekara et al., 2021). Concretely, each sample in the dataset consists of a dialog context, a generated response and a quality score. Optionally, a groundtruth response may also be included. Due to the space constraint, in this section we only provide a high-level overview of the used datasets. The complete list and discussion of characteristics of used datasets is provided in Appendix B.
In general, these datasets are constructed using the following steps:

1. Choose an existing dialog dataset.

2. Train a response generation model on the chosen dialog dataset.

3. Generate responses for the validation/test set of the chosen dataset

4. Collect human quality annotations for the generated responses.

For data that does not contain referenced responses, only reference-free metrics were assessed.

The characteristics of the quality-annotated data significantly influence the performance of the metrics since dialog metrics might be originally developed and trained on data in a very different domain than the test domain. For example, since many of these datasets were collected in different settings, they have diverse formality, complexity of the sentence structure, and the dialog length. Despite using the same underlying dialog dataset, the response generation model used would also influence the characteristics of responses. Distinguishing between responses with very different quality is easier, since the low quality response may not even follow grammar rules or the dialog context. On the other hand, it will be challenging to give appropriate scores to responses from state-of-the-art dialog systems. In the 10 datasets, responses in the data labeled by GRADE, FED, and DSTC9 data come from models with relatively better empirical performance such as Transformer Seq2Seq model (Vaswani et al., 2017), DialoGPT (Zhang et al., 2020), and Meena (Adiwardana et al., 2020b).

\section{Experiments}

This section describes the assessment of the evaluation metrics. Wherever possible, the released pretrained model was used to reproduce results. Since RUBER, BERT-RUBER and PONE do not release their pretrained models, those models were trained on DailyDialog. For USR, we use the released model which is trained on TopicalChat. For PredictiveEngage, the original paper combined the model with RUBER for the best performance. In this assessment only the engagement score model is used for comparison to the other metrics. FlowScore only scores dialogs with more than 3 utterances, so it cannot be used on some of the quality-annotated data. 


\begin{tabular}{l|cccc}
\hline Metric & Pretrained Model & Training Dataset & Reference-Free? & Objective \\
\hline BLEU (2002) & $\mathrm{X}$ & $\mathrm{X}$ & $\mathrm{X}$ & $\mathrm{X}$ \\
METEOR (2005) & $\mathrm{X}$ & $\mathrm{X}$ & $\mathrm{X}$ & $\mathrm{X}$ \\
ROUGE (2004) & $\mathrm{X}$ & $\mathrm{X}$ & $\mathrm{X}$ & $\mathrm{X}$ \\
ADEM (2017) & $\mathrm{X}$ & Ubuntu Dialogue + Twitter & $\mathrm{X}$ & MSE \\
BERTScore (2019) & BERT & $\mathrm{X}$ & $\mathrm{X}$ & $\mathrm{X}$ \\
BLEURT (2020) & BERT & WMT Metrics Shared Task & $\mathrm{X}$ & MSE \\
QuestEval (2021) & $\mathrm{T} 5$ & SQuAD-v2/NewsQA & $\sqrt{ }$ & QA/QG \\
RUBER (2018) & $\mathrm{X}$ & DailyDialog / PersonaChat & $\mathrm{X}$ & Triplet \\
BERT-RUBER (2019) & BERT & DailyDialog / PersonaChat & $\mathrm{X}$ & Triplet \\
PONE (2020) & BERT & DailyDialog & $\mathrm{X}$ & Triplet \\
MAUDE (2020) & BERT & PersonaChat & $\sqrt{ }$ & NCE \\
DEB (2020) & BERT & Reddit/DailyDialog++ & $\sqrt{ }$ & MLM/NSP \\
GRADE (2020) & BERT & DailyDialog & $\sqrt{ }$ & Triplet \\
DynaEval (2021a) & RoBERTa & ED/ConvA2/DailyDialog & $\sqrt{ }$ & Triplet \\
USR (2020b) & RoBERTa & TopicalChat/PersonaChat & $\sqrt{ }$ & MLM/CrossEntropy \\
USL-H (2020) & BERT & DailyDialog & $\sqrt{ }$ & VUP/NSP/MLM \\
DialogRPT (2020) & GPT-2 & Reddit & CrossEntropy \\
Deep AM-FM (2021b) & Multilingual BERT & Twitter & $\mathrm{X}$ & MLM \\
HolisticEval (2020) & BERT & DailyDialog & $\sqrt{ }$ & LM \\
PredictiveEngage (2020) & BERT & ConvAI & $X$ & CrossEntropy \\
FED (2020a) & DialoGPT & X & $\sqrt{ }$ & X \\
FlowScore (2021b) & Plato & Reddit & $\sqrt{ }$ & ContextFlow \\
FBD (2021) & RoBERTa & X & $X$ & X \\
& & & &
\end{tabular}

Table 1: Summary of the evaluation metrics assessed in this paper. The 'Pretrained Model' column indicates the specific pretrained language model used by the metric. The 'Training Dataset' and 'Objective' columns describe the dialog data and the objective used when training the metric. 'Reference-Free?' indicates whether the metric requires a reference response for evaluation. ED is the abbreviation of the EmpatheticDialogue dataset.

\subsection{Results on Datasets with Human Reference}

This section describes the performance of the metrics on the quality-annotated datasets that contain a human reference. This distinction is important, because the referenced metrics (e.g., BLEU, METEOR, BERTScore) can only be assessed on these datasets. Table 2 present the correlations of the metrics on the USR, GRADE, and DSTC6 data. Due to the space constraint, we only present Spearman correlation, and the full table could be found in Appendix C.

Rule-based metrics perform surprisingly well on USR-TopicalChat and USR-PersonaChat. However, they fall short on the GRADE data. This may be due to the fact that the responses in the GRADE data are produced by better NLG models, as described in Section 3. Since rule-based metrics calculate word-overlap metrics between the system responses and a human reference, they can consistently detect poor responses which only contain irrelevant words. However, the rule-based metrics struggle to score responses from state-of-the-art dialog systems since those responses require a deeper semantic understanding of the dialog context and the generated response.

USR, GRADE, DEB, and USL-H are the best performing metrics for evaluating response generation (i.e., turn-level correlation). The USR and GRADE metrics have high performance on their respective datasets. This phenomenon probably occurs for several reasons. First, both metrics finetune pretrained models in a self-supervised manner on certain dialog datasets. It is expected that the metrics would perform better on quality-annotated datasets corresponding to the data they were finetuned on. For example, the choice of the topic graph of GRADE may have been influenced by phenomena observed in the GRADE data. Finally, metrics may be optimized specifically for the data (i.e., through hyperparameter tuning on a validation set). This observation stresses the importance of testing on a variety of different quality-annotated datasets in order to ensure the generality of an evaluation metric and to avoid over-optimizing to specific datasets.

The performance of DEB demonstrates the importance of training data in model-based metrics. DEB replaces the BERT pretraining data with a dialog corpus, and further finetunes the model on a manually-created dataset consisting of adversarial responses designed for the NSP objective. USL$\mathrm{H}$ has a similar performance trend to USR, which is reasonable since the two metrics both combine response selection models and language models. The VUP model which validates whether a given response is grammatically correct helps USL-H outperforms USR on the USR-PersonaChat dataset.

\subsection{Results on Datasets without Human Reference}

This section describes the results for the datasets that do not contain a reference response. Only reference-free metrics can be evaluated on these quality-annotated datasets.

Table 3 presents results of the reference-free 


\begin{tabular}{|c|c|c|c|c|c|c|c|c|c|c|c|c|}
\hline & \multicolumn{2}{|c|}{ USR-TopicalChat } & \multicolumn{2}{|c|}{ USR-PersonaChat } & \multicolumn{2}{|c|}{ GRADE-ConvAI2 } & \multicolumn{2}{|c|}{ GRADE-DD } & \multicolumn{2}{|c|}{ GRADE-ED } & \multicolumn{2}{|c|}{ DSTC6 } \\
\hline & Turn & System & Turn & System & Turn & System & Turn & System & Turn & System & Turn & System \\
\hline BLEU-4 & 0.296 & 0.900 & $0.090^{*}$ & $0.800 *$ & 0.128 & $0.000 *$ & 0.184 & 1.000 & $0.002 *$ & 1.000 & 0.298 & $0.050^{*}$ \\
\hline METEOR & 0.391 & 0.900 & 0.271 & $0.800 *$ & 0.181 & $0.600^{*}$ & $0.010^{*}$ & -1.000 & $0.055^{*}$ & 1.000 & 0.323 & $0.084 *$ \\
\hline ROUGE-L & 0.287 & 0.900 & $0.038^{*}$ & $0.000 *$ & 0.140 & $0.000 *$ & 0.147 & 1.000 & $-0.013^{*}$ & 1.000 & 0.326 & $0.215^{*}$ \\
\hline ADEM & $-0.061 *$ & $0.700 *$ & $-0.085 *$ & $0.400 *$ & $-0.057 *$ & $-0.200 *$ & $0.071 *$ & 1.000 & $-0.028^{*}$ & 1.000 & 0.118 & $0.347 *$ \\
\hline BERTScore & 0.325 & 0.900 & $0.122 *$ & $0.000 *$ & 0.224 & $0.800^{*}$ & $0.100^{*}$ & -1.000 & $0.033^{*}$ & 1.000 & 0.337 & $0.265^{*}$ \\
\hline BLEURT & 0.261 & 0.900 & $0.054 *$ & $0.000 *$ & 0.120 & $-0.400^{*}$ & 0.133 & 1.000 & $0.051 *$ & 1.000 & 0.294 & $0.426^{*}$ \\
\hline QuestEval & 0.338 & 1.000 & 0.236 & 1.000 & 0.319 & $0.400 *$ & $0.006^{*}$ & -1.000 & 0.272 & 1.000 & 0.242 & $0.206^{*}$ \\
\hline RUBER & 0.259 & 1.000 & 0.190 & 1.000 & $-0.042 *$ & $-0.400 *$ & $-0.094 *$ & -1.000 & $-0.039^{*}$ & 1.000 & 0.092 & $0.104 *$ \\
\hline BERT-RUBER & 0.348 & 0.900 & 0.248 & $0.200 *$ & 0.314 & 1.000 & 0.128 & -1.000 & 0.148 & 1.000 & 0.217 & $0.093 *$ \\
\hline PONE & 0.274 & $0.500 *$ & 0.375 & $0.800 *$ & 0.373 & $0.800^{*}$ & 0.163 & -1.000 & 0.161 & 1.000 & 0.200 & $0.235 *$ \\
\hline MAUDE & $0.083 *$ & $-0.200 *$ & 0.298 & $0.400 *$ & 0.304 & $0.800 *$ & $-0.073 *$ & 1.000 & $-0.057^{*}$ & 1.000 & 0.128 & $0.217 *$ \\
\hline DEB & 0.116 & $0.400 *$ & 0.373 & 1.000 & 0.504 & 1.000 & 0.363 & 1.000 & 0.395 & 1.000 & 0.214 & 0.492 \\
\hline GRADE & 0.217 & $0.100 *$ & 0.352 & 1.000 & 0.571 & $0.800^{*}$ & 0.253 & -1.000 & 0.297 & 1.000 & 0.122 & 0.611 \\
\hline DynaEval & $-0.022 *$ & $0.100 *$ & 0.171 & $0.800^{*}$ & 0.131 & -1.000 & 0.120 & -1.000 & 0.141 & -1.000 & 0.246 & $-0.050 *$ \\
\hline USR & 0.423 & 0.900 & 0.418 & 1.000 & 0.500 & 1.000 & $0.057^{*}$ & -1.000 & 0.255 & 1.000 & 0.166 & $0.147^{*}$ \\
\hline USL-H & 0.340 & 0.900 & 0.523 & $0.800 *$ & 0.457 & 1.000 & $0.093 *$ & -1.000 & 0.235 & 1.000 & 0.179 & $0.298 *$ \\
\hline DialogRPT & $0.105^{*}$ & $0.600 *$ & $-0.083^{*}$ & $0.800 *$ & 0.158 & $-0.600^{*}$ & $0.037 *$ & -1.000 & 0.203 & 1.000 & 0.155 & $0.334 *$ \\
\hline Deep AM-FM & 0.268 & $0.700 *$ & 0.219 & 1.000 & 0.130 & $0.400^{*}$ & $0.022 *$ & 1.000 & $0.058^{*}$ & 1.000 & 0.295 & 0.674 \\
\hline HolisticEval & -0.123 & $-0.200 *$ & $0.113 *$ & $0.000 *$ & $-0.010^{*}$ & $-0.400 *$ & $0.020^{*}$ & 1.000 & 0.204 & -1.000 & $-0.004 *$ & -0.002 \\
\hline PredictiveEngage & 0.310 & 0.900 & $0.033^{*}$ & $0.200 *$ & 0.164 & $0.600^{*}$ & -0.135 & -1.000 & $-0.078^{*}$ & 1.000 & $0.004 *$ & $-0.409^{*}$ \\
\hline FED & -0.135 & $0.100 *$ & $-0.000^{*}$ & $0.400 *$ & $-0.072^{*}$ & $0.000^{*}$ & $0.064 *$ & 1.000 & $-0.044 *$ & 1.000 & -0.083 & $0.322 *$ \\
\hline FlowScore & $0.082 *$ & $0.400 *$ & $0.079 *$ & $0.800 *$ & - & - & - & - & - & - & 0.095 & $0.362 *$ \\
\hline FBD & - & $0.100 *$ & - & $0.800^{*}$ & - & $-0.400^{*}$ & - & -1.000 & - & -1.000 & - & $-0.234^{*}$ \\
\hline GRADE + DEB & 0.217 & $0.400 *$ & 0.403 & 1.000 & 0.549 & 1.000 & 0.325 & 1.000 & 0.391 & 1.000 & 0.200 & 0.501 \\
\hline $\mathrm{USR}$ + USL + GRADE + DEB & 0.382 & $0.700 *$ & 0.495 & 1.000 & 0.579 & 1.000 & 0.198 & -1.000 & 0.387 & 1.000 & 0.238 & 0.553 \\
\hline ALL & 0.473 & 0.900 & 0.534 & 1.000 & 0.561 & 1.000 & 0.149 & -1.000 & 0.349 & 1.000 & 0.268 & 0.761 \\
\hline
\end{tabular}

Table 2: Results on datasets with human reference. All values are statistically significant to $p<0.05$, unless marked by *. GRADE-DD and GRADE-ED indicate the GRADE-DailyDialog and GRADE-EmpatheticDialogue dataset, respectively. PE is the abbreviation of PredictiveEngage metric.

\begin{tabular}{|c|c|c|c|c|c|c|c|c|c|c|}
\hline & \multirow{2}{*}{\multicolumn{2}{|c|}{$\begin{array}{l}\text { PE-DailyDialog } \\
\text { Turn-Level }\end{array}$}} & \multicolumn{4}{|c|}{ FED } & \multicolumn{4}{|c|}{ DSTC9 } \\
\hline & & & \multicolumn{2}{|c|}{ Turn-Level } & \multicolumn{2}{|c|}{ Dialog-Level } & \multicolumn{2}{|c|}{ Dialog-Level } & \multicolumn{2}{|c|}{ System-Level } \\
\hline & $\mathrm{P}$ & $\mathrm{S}$ & $\mathrm{P}$ & $\mathrm{S}$ & $\mathrm{P}$ & $\mathrm{S}$ & $\mathrm{P}$ & $\mathrm{S}$ & $\mathrm{P}$ & $\mathrm{S}$ \\
\hline QuestEval & 0.296 & 0.341 & $0.037 *$ & $0.093^{*}$ & $-0.032 *$ & $0.080 *$ & $0.026 *$ & 0.043 & 0.604 & $0.527 *$ \\
\hline MAUDE & 0.104 & $0.060 *$ & $0.018 *$ & $-0.094 *$ & $-0.047 *$ & -0.280 & 0.059 & $0.042 *$ & $0.224 *$ & $0.045^{*}$ \\
\hline DEB & 0.516 & 0.580 & 0.230 & 0.187 & $-0.130 *$ & $0.006 *$ & 0.085 & 0.131 & 0.683 & $0.473 *$ \\
\hline GRADE & 0.600 & 0.622 & 0.134 & 0.118 & $-0.034 *$ & $-0.065^{*}$ & -0.078 & -0.070 & -0.674 & $-0.482 *$ \\
\hline DynaEval & 0.167 & 0.160 & 0.319 & 0.323 & 0.503 & 0.547 & 0.093 & 0.101 & 0.652 & 0.727 \\
\hline USR & 0.582 & 0.640 & 0.114 & 0.117 & $0.093 *$ & $0.062 *$ & $0.019^{*}$ & $0.020^{*}$ & $0.149^{*}$ & $0.127 *$ \\
\hline USL-H & 0.688 & 0.699 & 0.201 & 0.189 & $0.073^{*}$ & $0.152 *$ & 0.105 & 0.105 & $0.566^{*}$ & 0.755 \\
\hline DialogRPT & 0.489 & 0.533 & -0.118 & $-0.086 *$ & -0.221 & -0.214 & 0.076 & 0.069 & 0.685 & $0.555 *$ \\
\hline HolisticEval & 0.368 & 0.365 & 0.122 & 0.125 & -0.276 & -0.304 & $0.015^{*}$ & $0.002 *$ & $-0.019 *$ & $-0.100 *$ \\
\hline PredictiveEngage & 0.429 & 0.414 & $0.024 *$ & $0.094 *$ & $0.026 *$ & $0.155^{*}$ & 0.114 & 0.115 & 0.809 & 0.664 \\
\hline FED & 0.164 & 0.159 & 0.120 & 0.095 & 0.222 & 0.320 & 0.128 & 0.120 & $0.559^{*}$ & $0.391 *$ \\
\hline FlowScore & - & - & $-0.065^{*}$ & $-0.055^{*}$ & $-0.073^{*}$ & $-0.003^{*}$ & 0.147 & 0.140 & 0.907 & 0.900 \\
\hline FBD & 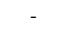 & 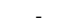 & . & 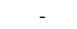 & (1) & 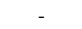 & rest & 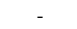 & -0.669 & -0.627 \\
\hline
\end{tabular}

Table 3: Results on PredictiveEngage-DailyDialog, FED, and DSTC9 data. P and S indicate Pearson correlation and Spearman correlation, respectively. Here, we denote PredictiveEngage-DailyDialog by PE-DailyDialog due to space constraint. All values are statistically significant to $p<0.05$, unless marked by *.

metrics on HolisticEval-DailyDialog, FED, and DSTC9 data.

In the FED dataset, there are two types of annotations: turn-level and dialog-level. Turn-level annotations assess the quality of a single response, while dialog-level annotations assess the entire dialog. Similar to the GRADE data, the responses in the FED data are generated by state-of-the-art dialog systems (Adiwardana et al., 2020b), which makes the data particularly challenging.

Many metrics which perform well on the USR and GRADE data, do not do as well on the FED data. This may be because of the longer dialog context in the FED data. While the average number of words in the context of GRADE-ConvAI2 is 23.4, the context of FED has on average 86.5 words and 11.8 utterances. If a model has not seen long contexts at training time, it will struggle to perform well on longer contexts at test time.

FlowScore, PredictiveEngage, DynaEval and FED perform the best on dialog-level evaluation on the FED and DSTC9 datasets. This may occur for two reasons. First, these models are designed to capture properties that are better aligned with dialog-level annotations: FlowScore models the dynamic infor- mation flow in the dialog history, DynaEval measures coherence using a graph-based representation of the dialog, PredictiveEngage measures engagement, FED measures a number of different dialog-level qualities (e.g., coherence, topic depth, etc.). Second, the model architectures of PredictiveEngage and FED are relatively simple. This may make the two metrics less sensitive to longer dialog contexts. Though these metrics do well at assessing state-of-the-art dialog systems in the FED and DSTC9 data, they underperform on 
the GRADE and USR data. This suggests that these two metrics are optimal for dialog-level evaluation, but less so for turn-level evaluation.

\subsection{Results on System-Level Correlation}

This section presents system-level correlation on quality-annotated datasets. System-level correlation is a strong indication of the ability of metrics to rank several response generation models. GRADEDailyDialog and GRADE-EmpatheticDialog only consist of 2 systems, which makes system-level correlation much less informative.

In general, metrics that have a higher turn-level correlation tend to have a higher system-level correlation. Interestingly, some metrics which perform poorly on turn-level correlation do much better on system-level correlation, suggesting that averaging out over all the examples produced by a dialog system reduces the noise in the metric's scores. For example, GRADE has a comparable system-level correlation to the best-performing Deep AM-FM on the DSTC6 data, despite having a lower turnlevel correlation. On the other hand, BERTScore, which has the highest turn-level correlation on the DSTC6 data, performs poorly on system-level correlation. This suggests that word-overlap metrics may struggle to accurately assess entire systems.

System-level correlations are generally very strong across a variety of metrics. Most impressively are the high correlations of Deep AM-FM on the DSTC6 data (11 different systems) and of FlowScore on the DSTC9 data (20 different systems).

\subsection{Performance on Various Dialog Qualities}

Since dialog quality is inherently multi-faceted (Walker et al., 1997; See et al., 2019), it is inadequate to only evaluate dialog metrics on the overall response score. Therefore, this paper also presents the correlation of the metrics with various dialog qualities in Table 4 and Figure 1.

HolisticEval-DailyDialog annotates the context coherence of responses. For the FED data, we present results on 8 fine-grained turn-level qualitites, and results on dialog-level qualities could be found in Appendix. For metrics that produce fine-grained scores, we use the corresponding finegrained score to measure correlation. For example, because PredictiveEngage evaluates the engaging quality of the dialog, the engaging score, rather than the overall score, is used to measure correlation with the engaging quality in the FED dataset.
USR and USL-H both do well predicting context coherence and engaging on the HolisticEvalDailyDialog and FED data, respectively. These two metrics also have good performance on the USR and GRADE data. In contrast, HolisticEval and PredictiveEngage do well on measuring coherence and engagement, but underperform on the USR and GRADE data. This suggests that while finegrained metrics are important qualities to measure, modelling only these qualities is insufficient. Instead, it is better to design a metric that measures many different qualities and can aggregate them to form an overall score, such as the combination of MLM/dialog retrieval in USR and MLM/VUP/NSP in USL-H. The evaluation of specific fine-grained qualities sheds much light on their underlying behavior.

\begin{tabular}{lcc}
\hline & \multicolumn{2}{c}{ HolisticEval-DailyDialog } \\
& Pearson & Spearman \\
\hline QuestEval & 0.285 & 0.260 \\
MAUDE & 0.275 & 0.364 \\
DEB & 0.584 & 0.663 \\
GRADE & $\mathbf{0 . 6 7 8}$ & 0.697 \\
DynaEval & $-0.023^{*}$ & $-0.009^{*}$ \\
USR & 0.589 & 0.645 \\
USL-H & 0.486 & 0.537 \\
DialogRPT & 0.283 & 0.332 \\
HolisticEval & 0.670 & $\mathbf{0 . 7 6 4}$ \\
PredictiveEngage & $-0.033^{*}$ & $0.060^{*}$ \\
FED & 0.485 & 0.507 \\
FlowScore & - & - \\
FBD & - & -
\end{tabular}

Table 4: Results on HolisticEval-DailyDialog. All values are statistically significant to $p<0.05$, unless marked by *.

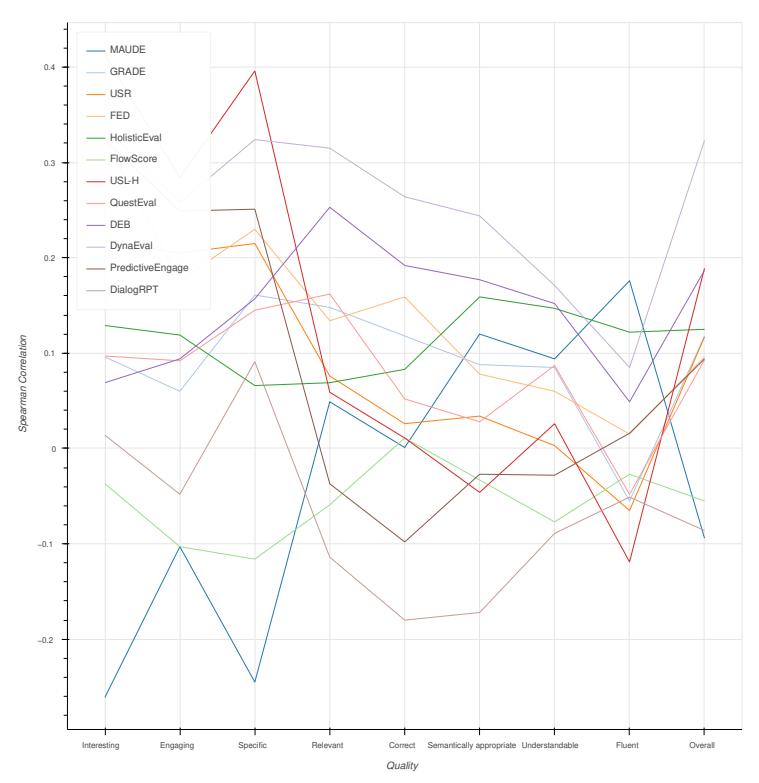

Figure 1: Spearman correlation to different turn-level 19 annotation qualities on the FED data. 




Figure 2: Spearman correlation when varying the dialog context length.

\subsection{Effect of Context Length}

Most reference-free metrics compute their scores by comparing the dialog context with the response. Therefore, it is interesting to determine if these metrics perform differently according to the length of the dialog context. The quality-annotated samples are grouped by their context lengths with a length interval of 10. Figure 2 shows performance differences of the reference-free metrics at different context lengths.

Many metrics' performance decreases as the context lengthens. This may be due to the fact that those metrics are trained on shorter dialogs and struggle to understand longer dialog contexts. On the other hand, the performance of HolisticEval, DialogRPT, and FED increases as the context lengthens. These metrics incorporate GPT-2-based language models to score responses, while the other metrics mostly rely on BERT-based models. BERTbased models are optimized for local coherence on the BookCorpus and Wikipedia through the MLM objective and limit context length. Thus GPT-2based metrics perform better on longer dialogs.

The performance of USL-H, DEB, and PredictiveEngage does not change much at different context lengths. For PredictiveEngage, this could be because PredictiveEngage uses several MLP layers during the pooling of the pretrained BERT embeddings to predict the output. USL-H uses the VUP model which aims to determine if a response is grammatically correct. The use of the VUP model may make USL-H robust to longer context lengths.
The BERT model used in DEB is pretrained on the Reddit corpus which may improve its ability to model long-range correlations in the dialog. These different factors make metrics more robust to different context lengths.

\subsection{Combining Metrics}

Many metrics rely on an ensemble of different models. Inspired by this, this section explores the possibility that a combination of metrics may be successful (Table 2). Since it is not feasible to exhaustively explore all possible combinations of the metrics, some combinations of the best-performing metrics were explored here. The metrics are combined through simple averaging. Future work should explore more sophisticated mechanisms for combining metrics.

In the last three rows of Table 2, we selectively present the three combinations of metrics with the best performance. The complete table and discussion could be found in Appendix C.3. In general, combinations of the best performing metrics USR, GRADE, USL-H, and DEB improve the performance. The last row shows results of taking the average of the scores of all of the metrics that were assessed in this paper. While the idea of an All metric is simple, and metrics are combined through simple averaging, the results are surprisingly good across the different referenced quality-annotated datasets. This result highlights the potential of combining various evaluation metrics in better manners.

\section{Conclusions}

This paper provides a comprehensive assessment of various automatic evaluation metrics for dialog. Many different evaluation metrics have been proposed in recent years. The results indicate several important directions for future work on the assessment of dialog evaluation metrics: 1) Many metrics rely on pretrained language models, and the analysis in this paper highlights the impacts of them. Researchers could further explore the impact of choice of pretrianed models. 2) Unsurprisingly, Metrics generally perform better on the data they were trained on. Therefore, methods to adapt metrics to new domains should be an important research direction. 3) Simple combinations of metrics yield promising results. Future work should explore more sophisticated ways for combining metrics. 


\section{Acknowledgements}

We thank the authors of the various metrics for releasing their code. This work is funded by National Science Foundation grant CNS-1512973. The opinions expressed in this paper do not necessarily reflect those of the National Science Foundation.

\section{References}

D. Adiwardana, Minh-Thang Luong, David R. So, J. Hall, Noah Fiedel, R. Thoppilan, Z. Yang, Apoorv Kulshreshtha, Gaurav Nemade, Yifeng Lu, and Quoc V. Le. 2020a. Towards a human-like opendomain chatbot. ArXiv, abs/2001.09977.

Daniel Adiwardana, Minh-Thang Luong, David R So, Jamie Hall, Noah Fiedel, Romal Thoppilan, Zi Yang, Apoorv Kulshreshtha, Gaurav Nemade, Yifeng Lu, et al. 2020b. Towards a human-like open-domain chatbot. arXiv preprint arXiv:2001.09977.

R Ananthakrishnan, Pushpak Bhattacharyya, M Sasikumar, and Ritesh M Shah. 2007. Some issues in automatic evaluation of english-hindi mt: more blues for bleu. ICON.

Satanjeev Banerjee and Alon Lavie. 2005. METEOR: An automatic metric for MT evaluation with improved correlation with human judgments. In Proceedings of the ACL Workshop on Intrinsic and Extrinsic Evaluation Measures for Machine Translation and/or Summarization, pages 65-72, Ann Arbor, Michigan. Association for Computational Linguistics.

Siqi Bao, Huang He, Fan Wang, Hua Wu, and Haifeng Wang. 2020. PLATO: Pre-trained dialogue generation model with discrete latent variable. In Proceedings of the 58th Annual Meeting of the Association for Computational Linguistics, pages 85-96, Online. Association for Computational Linguistics.

Kyunghyun Cho, Bart van Merriënboer, Caglar Gulcehre, Dzmitry Bahdanau, Fethi Bougares, Holger Schwenk, and Yoshua Bengio. 2014. Learning phrase representations using RNN encoder-decoder for statistical machine translation. In Proceedings of the 2014 Conference on Empirical Methods in Natural Language Processing (EMNLP), pages 17241734, Doha, Qatar. Association for Computational Linguistics.

Jan Deriu, Alvaro Rodrigo, Arantxa Otegi, Guillermo Echegoyen, Sophie Rosset, Eneko Agirre, and Mark Cieliebak. 2021. Survey on evaluation methods for dialogue systems. Artificial Intelligence Review, 54(1):755-810.

Jan Deriu, Don Tuggener, Pius von Däniken, Jon Ander Campos, Alvaro Rodrigo, Thiziri Belkacem, Aitor Soroa, Eneko Agirre, and Mark Cieliebak. 2020. Spot the bot: A robust and efficient framework for the evaluation of conversational dialogue systems. In Proceedings of the 2020 Conference on Empirical Methods in Natural Language Processing (EMNLP), pages 3971-3984, Online. Association for Computational Linguistics.

Jacob Devlin, Ming-Wei Chang, Kenton Lee, and Kristina Toutanova. 2019. BERT: Pre-training of deep bidirectional transformers for language understanding. In Proceedings of the 2019 Conference of the North American Chapter of the Association for Computational Linguistics: Human Language Technologies, Volume 1 (Long and Short Papers), pages 4171-4186, Minneapolis, Minnesota. Association for Computational Linguistics.

Emily Dinan, Varvara Logacheva, Valentin Malykh, Alexander Miller, Kurt Shuster, Jack Urbanek, Douwe Kiela, Arthur Szlam, Iulian Serban, Ryan Lowe, et al. 2019. The second conversational intelligence challenge (convai2). arXiv preprint arXiv:1902.00098.

Michel Galley, Chris Brockett, Alessandro Sordoni, Yangfeng Ji, Michael Auli, Chris Quirk, Margaret Mitchell, Jianfeng Gao, and Bill Dolan. 2015. deltaBLEU: A discriminative metric for generation tasks with intrinsically diverse targets. In Proceedings of the 53rd Annual Meeting of the Association for Computational Linguistics and the 7th International Joint Conference on Natural Language Processing (Volume 2: Short Papers), pages 445-450, Beijing, China. Association for Computational Linguistics.

Xiang Gao, Yizhe Zhang, Michel Galley, Chris Brockett, and Bill Dolan. 2020. Dialogue response rankingtraining with large-scale human feedback data. In $E M N L P$.

Sarik Ghazarian, Johnny Wei, Aram Galstyan, and Nanyun Peng. 2019. Better automatic evaluation of open-domain dialogue systems with contextualized embeddings. In Proceedings of the Workshop on Methods for Optimizing and Evaluating Neural Language Generation, pages 82-89, Minneapolis, Minnesota. Association for Computational Linguistics.

Sarik Ghazarian, Ralph Weischedel, Aram Galstyan, and Nanyun Peng. 2020. Predictive engagement: An efficient metric for automatic evaluation of opendomain dialogue systems. In Proceedings of the AAAI Conference on Artificial Intelligence, volume 34, pages 7789-7796.

Karthik Gopalakrishnan, Behnam Hedayatnia, Qinlang Chen, Anna Gottardi, Sanjeev Kwatra, Anu Venkatesh, Raefer Gabriel, and Dilek HakkaniTür. 2019. Topical-Chat: Towards KnowledgeGrounded Open-Domain Conversations. In Proc. Interspeech 2019, pages 1891-1895.

Jian Guan and Minlie Huang. 2020. UNION: An Unreferenced Metric for Evaluating Open-ended Story Generation. In Proceedings of the 2020 Conference 
on Empirical Methods in Natural Language Processing (EMNLP), pages 9157-9166, Online. Association for Computational Linguistics.

Chulaka Gunasekara, Seokhwan Kim, Luis Fernando D'Haro, Abhinav Rastogi, Yun-Nung Chen, Mihail Eric, Behnam Hedayatnia, Karthik Gopalakrishnan, Yang Liu, Chao-Wei Huang, et al. 2021. Overview of the ninth dialog system technology challenge: Dstc9. Proceedings of the 9th Dialog System Technology Challenge Workshop in AAAI2021.

Fenfei Guo, A. Metallinou, Chandra Khatri, Anirudh Raju, Anu Venkatesh, and A. Ram. 2018. Topicbased evaluation for conversational bots. ArXiv, abs/1801.03622.

Michael Gutmann and Aapo Hyvärinen. 2010. Noisecontrastive estimation: A new estimation principle for unnormalized statistical models. In Proceedings of the Thirteenth International Conference on Artificial Intelligence and Statistics, pages 297-304. JMLR Workshop and Conference Proceedings.

Chiori Hori and Takaaki Hori. 2017. Endto-end conversation modeling track in dstc6. arXiv: 1706.07440 .

Lishan Huang, Zheng Ye, Jinghui Qin, Liang Lin, and Xiaodan Liang. 2020. GRADE: Automatic graphenhanced coherence metric for evaluating opendomain dialogue systems. In Proceedings of the 2020 Conference on Empirical Methods in Natural Language Processing (EMNLP), pages 9230-9240, Online. Association for Computational Linguistics.

Ryan Kiros, Yukun Zhu, Ruslan Salakhutdinov, Richard S Zemel, Antonio Torralba, Raquel Urtasun, and Sanja Fidler. 2015. Skip-thought vectors. arXiv preprint arXiv:1506.06726.

Tian Lan, Xian-Ling Mao, Wei Wei, Xiaoyan Gao, and Heyan Huang. 2020. Pone: A novel automatic evaluation metric for open-domain generative dialogue systems. ACM Trans. Inf. Syst., 39(1).

Jiwei Li, Michel Galley, Chris Brockett, Jianfeng Gao, and Bill Dolan. 2016. A diversity-promoting objective function for neural conversation models. In Proceedings of the 2016 Conference of the North American Chapter of the Association for Computational Linguistics: Human Language Technologies, pages 110-119, San Diego, California. Association for Computational Linguistics.

Yanran Li, Hui Su, Xiaoyu Shen, Wenjie Li, Ziqiang Cao, and Shuzi Niu. 2017. DailyDialog: A manually labelled multi-turn dialogue dataset. In Proceedings of the Eighth International Joint Conference on Natural Language Processing (Volume 1: Long Papers), pages 986-995, Taipei, Taiwan. Asian Federation of Natural Language Processing.

Zekang Li, Jinchao Zhang, Zhengcong Fei, Yang Feng, and Jie Zhou. 2021a. Addressing inquiries about history: An efficient and practical framework for evaluating open-domain chatbot consistency. In Findings of Proceedings of the 59th Annual Meeting of the Association for Computational Linguistics.

Zekang Li, Jinchao Zhang, Zhengcong Fei, Yang Feng, and Jie Zhou. 2021b. Conversations are not flat: Modeling the intrinsic information flow between dialogue utterances. In Proceedings of the 59th Annual Meeting of the Association for Computational Linguistics.

Chin-Yew Lin. 2004. ROUGE: A package for automatic evaluation of summaries. In Text Summarization Branches Out, pages 74-81, Barcelona, Spain. Association for Computational Linguistics.

Chia-Wei Liu, Ryan Lowe, Iulian Serban, Mike Noseworthy, Laurent Charlin, and Joelle Pineau. 2016a. How NOT to evaluate your dialogue system: An empirical study of unsupervised evaluation metrics for dialogue response generation. In Proceedings of the 2016 Conference on Empirical Methods in Natural Language Processing, pages 2122-2132, Austin, Texas. Association for Computational Linguistics.

Chia-Wei Liu, Ryan Lowe, Iulian V Serban, Michael Noseworthy, Laurent Charlin, and Joelle Pineau. 2016b. How not to evaluate your dialogue system: An empirical study of unsupervised evaluation metrics for dialogue response generation. arXiv preprint arXiv:1603.08023.

Yinhan Liu, Myle Ott, Naman Goyal, Jingfei Du, Mandar Joshi, Danqi Chen, Omer Levy, Mike Lewis, Luke Zettlemoyer, and Veselin Stoyanov. 2019. Roberta: A robustly optimized bert pretraining approach. arXiv preprint arXiv: 1907.11692.

Ryan Lowe, Michael Noseworthy, Iulian Vlad Serban, Nicolas Angelard-Gontier, Yoshua Bengio, and Joelle Pineau. 2017. Towards an automatic Turing test: Learning to evaluate dialogue responses. In Proceedings of the 55th Annual Meeting of the Association for Computational Linguistics (Volume 1: Long Papers), pages 1116-1126, Vancouver, Canada. Association for Computational Linguistics.

Ryan Lowe, Nissan Pow, Iulian Serban, and Joelle Pineau. 2015. The Ubuntu dialogue corpus: A large dataset for research in unstructured multi-turn dialogue systems. In Proceedings of the 16th Annual Meeting of the Special Interest Group on Discourse and Dialogue, pages 285-294, Prague, Czech Republic. Association for Computational Linguistics.

Joel Mackenzie, Rodger Benham, Matthias Petri, Johanne R Trippas, J Shane Culpepper, and Alistair Moffat. 2020. Cc-news-en: A large english news corpus. In Proceedings of the 29th ACM International Conference on Information \& Knowledge Management, pages 3077-3084.

Shikib Mehri and Maxine Eskenazi. 2020a. Unsupervised evaluation of interactive dialog with $\mathrm{Di}$ aloGPT. In Proceedings of the 21th Annual Meeting of the Special Interest Group on Discourse and 
Dialogue, pages 225-235, 1st virtual meeting. Association for Computational Linguistics.

Shikib Mehri and Maxine Eskenazi. 2020b. USR: An unsupervised and reference free evaluation metric for dialog generation. In Proceedings of the 58th Annual Meeting of the Association for Computational Linguistics, pages 681-707, Online. Association for Computational Linguistics.

Rostislav Nedelchev, Jens Lehmann, and Ricardo Usbeck. 2020. Language model transformers as evaluators for open-domain dialogues. In Proceedings of the 28th International Conference on Computational Linguistics, pages 6797-6808, Barcelona, Spain (Online). International Committee on Computational Linguistics.

Jekaterina Novikova, Ondřej Dušek, Amanda Cercas Curry, and Verena Rieser. 2017. Why we need new evaluation metrics for NLG. In Proceedings of the 2017 Conference on Empirical Methods in Natural Language Processing, pages 2241-2252, Copenhagen, Denmark. Association for Computational Linguistics.

Bo Pang, Erik Nijkamp, Wenjuan Han, Linqi Zhou, Yixian Liu, and Kewei Tu. 2020. Towards holistic and automatic evaluation of open-domain dialogue generation. In Proceedings of the 58th Annual Meeting of the Association for Computational Linguistics, pages 3619-3629, Online. Association for Computational Linguistics.

Kishore Papineni, Salim Roukos, Todd Ward, and WeiJing Zhu. 2002. Bleu: a method for automatic evaluation of machine translation. In Proceedings of the 40th Annual Meeting of the Association for Computational Linguistics, pages 311-318, Philadelphia, Pennsylvania, USA. Association for Computational Linguistics.

Vitou Phy, Yang Zhao, and Akiko Aizawa. 2020 Deconstruct to reconstruct a configurable evaluation metric for open-domain dialogue systems. In Proceedings of the 28th International Conference on Computational Linguistics, pages 4164-4178, Barcelona, Spain (Online). International Committee on Computational Linguistics.

Alec Radford, Jeffrey Wu, Rewon Child, David Luan, Dario Amodei, and Ilya Sutskever. 2019. Language models are unsupervised multitask learners. OpenAI blog, 1(8):9.

Colin Raffel, Noam Shazeer, Adam Roberts, Katherine Lee, Sharan Narang, Michael Matena, Yanqi Zhou, Wei Li, and Peter J. Liu. 2020. Exploring the limits of transfer learning with a unified text-totext transformer. Journal of Machine Learning Research, 21(140):1-67.

Hannah Rashkin, Eric Michael Smith, Margaret Li, and Y-Lan Boureau. 2019. Towards empathetic opendomain conversation models: A new benchmark and dataset. In Proceedings of the 57th Annual Meeting of the Association for Computational Linguistics, pages 5370-5381, Florence, Italy. Association for Computational Linguistics.

Cl'ement Rebuffel, Thomas Scialom, Laure Soulier, Benjamin Piwowarski, Sylvain Lamprier, Jacopo Staiano, Geoffrey Scoutheeten, and P. Gallinari. 2021. Data-questeval: A referenceless metric for data to text semantic evaluation. ArXiv, abs/2104.07555.

Ehud Reiter. 2018. A structured review of the validity of BLEU. Computational Linguistics, 44(3):393401.

Alan Ritter, Colin Cherry, and William B. Dolan. 2011. Data-driven response generation in social media. In Proceedings of the 2011 Conference on Empirical Methods in Natural Language Processing, pages 583-593, Edinburgh, Scotland, UK. Association for Computational Linguistics.

Mario Rodríguez-Cantelar, Luis Fernando D'Haro, and Fernando Matía. 2021. Automatic evaluation of nontask oriented dialog systems by using sentence embeddings projections and their dynamics. In Conversational Dialogue Systems for the Next Decade, pages 71-84. Springer.

Ananya B. Sai, Akash Kumar Mohankumar, Siddhartha Arora, and Mitesh M. Khapra. 2020. Improving dialog evaluation with a multi-reference adversarial dataset and large scale pretraining. Transactions of the Association for Computational Linguistics, 8:810-827.

Thomas Scialom, Paul-Alexis Dray, Patrick Gallinari, Sylvain Lamprier, Benjamin Piwowarski, Jacopo Staiano, and Alex Wang. 2021. Questeval: Summarization asks for fact-based evaluation. arXiv preprint arXiv:2103.12693.

Abigail See, Stephen Roller, Douwe Kiela, and Jason Weston. 2019. What makes a good conversation? how controllable attributes affect human judgments. In Proceedings of the 2019 Conference of the North American Chapter of the Association for Computational Linguistics: Human Language Technologies, Volume 1 (Long and Short Papers), pages 1702-1723, Minneapolis, Minnesota. Association for Computational Linguistics.

Thibault Sellam, Dipanjan Das, and Ankur Parikh. 2020. BLEURT: Learning robust metrics for text generation. In Proceedings of the 58th Annual Meeting of the Association for Computational Linguistics, pages 7881-7892, Online. Association for Computational Linguistics.

Hiroki Shimanaka, Tomoyuki Kajiwara, and Mamoru Komachi. 2018. RUSE: Regressor using sentence embeddings for automatic machine translation evaluation. In Proceedings of the Third Conference on Machine Translation: Shared Task Papers, pages 
751-758, Belgium, Brussels. Association for Computational Linguistics.

Koustuv Sinha, Prasanna Parthasarathi, Jasmine Wang, Ryan Lowe, William L. Hamilton, and Joelle Pineau. 2020. Learning an unreferenced metric for online dialogue evaluation. In Proceedings of the 58th Annual Meeting of the Association for Computational Linguistics, pages 2430-2441, Online. Association for Computational Linguistics.

Elior Sulem, Omri Abend, and Ari Rappoport. 2018. BLEU is not suitable for the evaluation of text simplification. In Proceedings of the 2018 Conference on Empirical Methods in Natural Language Processing, pages 738-744, Brussels, Belgium. Association for Computational Linguistics.

Chongyang Tao, Lili Mou, Dongyan Zhao, and Rui Yan. 2018. Ruber: An unsupervised method for automatic evaluation of open-domain dialog systems. In Proceedings of the AAAI Conference on Artificial Intelligence, volume 32.

Ashish Vaswani, Noam Shazeer, Niki Parmar, Jakob Uszkoreit, Llion Jones, Aidan N Gomez, Łukasz Kaiser, and Illia Polosukhin. 2017. Attention is all you need. In Advances in neural information processing systems, pages 5998-6008.

Anu Venkatesh, Chandra Khatri, Ashwin Ram, Fenfei Guo, Raefer Gabriel, Ashish Nagar, Rohit Prasad, Ming Cheng, Behnam Hedayatnia, Angeliki Metallinou, et al. 2018. On evaluating and comparing conversational agents. arXiv preprint arXiv:1801.03625, 4:60-68.

Marilyn A. Walker, Diane J. Litman, Candace A. Kamm, and Alicia Abella. 1997. PARADISE: A framework for evaluating spoken dialogue agents. In 35th Annual Meeting of the Association for Computational Linguistics and 8th Conference of the European Chapter of the Association for Computational Linguistics, pages 271-280, Madrid, Spain. Association for Computational Linguistics.

Alex Wang, Amanpreet Singh, Julian Michael, Felix Hill, Omer Levy, and Samuel Bowman. 2018. GLUE: A multi-task benchmark and analysis platform for natural language understanding. In Proceedings of the 2018 EMNLP Workshop BlackboxNLP: Analyzing and Interpreting Neural Networks for NLP, pages 353-355, Brussels, Belgium. Association for Computational Linguistics.

Jiannan Xiang, Yahui Liu, Deng Cai, Huayang Li, Defu Lian, and Lemao Liu. 2021. Assessing dialogue systems with distribution distances. arXiv preprint arXiv:2105.02573.

Tsuta Yuma, Naoki Yoshinaga, and Masashi Toyoda. 2020. uBLEU: Uncertainty-aware automatic evaluation method for open-domain dialogue systems. In Proceedings of the 58th Annual Meeting of the Association for Computational Linguistics: Student Research Workshop, pages 199-206, Online. Association for Computational Linguistics.
Chen Zhang, Yiming Chen, Luis Fernando D'Haro, Yan Zhang, Thomas Friedrichs, Grandee Lee, and Haizhou Li. 2021a. Dynaeval: Unifying turn and dialogue level evaluation. In The Joint Conference of the 59th Annual Meeting of the Association for Computational Linguistics and the 11th International Joint Conference on Natural Language Processing (ACL-IJCNLP 2021), Online.

Chen Zhang, Luis Fernando D'Haro, Rafael E Banchs, Thomas Friedrichs, and Haizhou Li. 2021b. Deep am-fm: Toolkit for automatic dialogue evaluation. In Conversational Dialogue Systems for the Next Decade, pages 53-69. Springer.

Saizheng Zhang, Emily Dinan, Jack Urbanek, Arthur Szlam, Douwe Kiela, and Jason Weston. 2018. Personalizing dialogue agents: I have a dog, do you have pets too? In Proceedings of the 56th Annual Meeting of the Association for Computational Linguistics (Volume 1: Long Papers), pages 2204 2213, Melbourne, Australia. Association for Computational Linguistics.

Tianyi Zhang, Varsha Kishore, Felix Wu, Kilian Q Weinberger, and Yoav Artzi. 2019. Bertscore: Evaluating text generation with bert. arXiv preprint arXiv:1904.09675.

Yizhe Zhang, Siqi Sun, Michel Galley, Yen-Chun Chen, Chris Brockett, Xiang Gao, Jianfeng Gao, Jingjing Liu, and Bill Dolan. 2020. Dialogpt: Large-scale generative pre-training for conversational response generation. In ACL, system demonstration.

Tiancheng Zhao, Ran Zhao, and Maxine Eskenazi. 2017a. Learning discourse-level diversity for neural dialog models using conditional variational autoencoders. In Proceedings of the 55th Annual Meeting of the Association for Computational Linguistics (Volume 1: Long Papers), pages 654-664, Vancouver, Canada. Association for Computational Linguistics.

Tiancheng Zhao, Ran Zhao, and Maxine Eskenazi. 2017b. Learning discourse-level diversity for neural dialog models using conditional variational autoencoders. arXiv preprint arXiv:1703.10960.

Wangchunshu Zhou and Ke Xu. 2020. Learning to compare for better training and evaluation of open domain natural language generation models. In Proceedings of the AAAI Conference on Artificial Intelligence, volume 34, pages 9717-9724.

Yukun Zhu, Ryan Kiros, Rich Zemel, Ruslan Salakhutdinov, Raquel Urtasun, Antonio Torralba, and Sanja Fidler. 2015. Aligning books and movies: Towards story-like visual explanations by watching movies and reading books. In Proceedings of the IEEE international conference on computer vision, pages 1927. 


\section{A Details of Studied Automatic Metrics}

\section{A.1 Design of the Metrics in this Assessment}

In general, dialog evaluation metrics can be divided into rule-based and model-based metrics. Rulebased metrics use heuristic rules to evaluate the system response, conditioned on the dialog context and human reference(s). Model-based metrics are trained, often with self-supervised objectives, to measure the quality of the responses.

BLEU (Papineni et al., 2002) is a popular rulebased metric often used to benchmark natural language generation (NLG) systems. BLEU computes the n-gram precision of the system responses using human references. While BLEU performs reasonably when evaluating NLG systems, it has issues reflecting grammaticality, use of semantically similar words, and meaning preservation (Novikova et al., 2017; Ananthakrishnan et al., 2007; Sulem et al., 2018; Reiter, 2018).

METEOR (Banerjee and Lavie, 2005) and ROUGE (Lin, 2004) have been proposed to address the shortcomings of BLEU. METEOR incorporates stems and synonyms into its calculation, while ROUGE focuses on n-gram recall instead of precision. Although these two metrics improve upon BLEU, they remain ineffective for dialog evaluation (Liu et al., 2016b). In general, word-overlap metrics struggle to evaluate dialog responses because of the one-to-many nature of dialog (Zhao et al., 2017b). Concretely, as mentioned above, since there are many appropriate responses for a given dialog context it is unreasonable to penalize a valid system response that deviates from the ground truth.

ADEM (Lowe et al., 2017) is an early learningbased metric that uses a recurrent neural network (RNN) to predict the quality of system responses. ADEM uses quality-annotated training data and the model is trained to predict human quality annotations with a mean squared error loss (MSE).

RUBER (Tao et al., 2018) uses a hybrid model consisting of both a referenced metric and an unreferenced metric. The referenced metric calculates the cosine similarity of word embeddings between a system response and a human reference. The unreferenced metric is trained with a triplet ranking loss to predict whether the generated response is appropriate for the dialog history. BERT-RUBER (Ghazarian et al., 2019) proposes to replace the RNN in RUBER with BERT (Devlin et al., 2019) to further improve the performance with contextualized word embeddings.

Based on BERT-RUBER, PONE (Lan et al., 2020) uses a novel algorithm to sample negative examples during training, and trains the metric on a dataset augmented by other NLG models.

MAUDE (Sinha et al., 2020) is trained with Noise Contrastive Estimation (NCE) (Gutmann and Hyvärinen, 2010) which requires the model to differentiate between a correct response and randomly sampled negative responses.

DEB (Sai et al., 2020) constructs a dialog dataset which consists of manually-created relevant responses and adversarial irrelevant responses. DEB uses BERT for dialog evaluation by first pretraining on a large-scale dialog corpus, and then fine-tuning on the proposed dataset with a next sentence prediction (NSP) objective.

GRADE (Huang et al., 2020) models topic transition dynamics in dialog by constructing a graph representation of the dialog history. This graph is then passed as input to a model that is trained with the same triplet loss as RUBER.

While GRADE is focused on turn-level topic transition dynamics in dialog, DynaEval (Zhang et al., 2021a) leverages a graph structure to model the dialog-level interactions between a user and a system. Through this graph-based approach, DynaEval is trained to distinguish well-formed dialogs from carefully constructed negative samples.

USR (Mehri and Eskenazi, 2020b) trains several models to measure different qualities of dialogs. USR relies on three different models, (1) a language model, trained with the masked language modelling (MLM) objectives, measures fluency, (2) a dialog retrieval model determines the relevance of a response and (3) a fact-to-response selection model measures whether a response conditions on knowledge.

Similarly, USL-H (Phy et al., 2020) combines three models trained with different objectives: valid utterance prediction (VUP), next sentence prediction (NSP), and MLM. The VUP model determines whether a response is valid and grammatically correct. The NSP model and MLM models are trained with self-supervised objectives to evaluate the sensibleness and the likelihood of a given response.

DialogRPT (Gao et al., 2020) is an ensemble of multiple GPT- 2 based models, which were finetuned on the Reddit human feedback data with different tasks. The tasks include predicting human feedback of responses and whether the response is 


\begin{tabular}{ll}
\hline Metrics & Reason for not using \\
\hline UNION (2020) & Designed for story generation \\
Embedding Methods (2015; 2016a; 2021) & Computing the embedding similarity is identical to the referenced metric in RUBER. \\
Language Model Evaluator (2020) & Using the language model probability is identical to the approach of USR and HolisticEval. \\
Perplexity (2020a) & No access to the NLG models that generated the quality-annotated data in order to obtain perplexity. \\
Distinct N-grams (2016) & This is not feasible for models trained on dialog corpora (Pang et al., 2020). \\
Spot The Bot (2020) & This still needs human annotation when evaluating models. \\
Learning to Compare (2020) & No released code, data, or pretrained models. \\
RUSE (2018) & Designed for machine translation only. \\
uBLEU (2020) & No released code. \\
deltaBLEU (2015) & For each response, this requires multiple human references to calculate the score. \\
Data-QuestEval (2021) & Designed for general NLU. not specifically for dialog. \\
Topic-based Evaluation (2018) & No released code, data, or pretrained models. \\
The Alexa Prize Evaluation Framework (2018) & No released code, data, or pretrained models. \\
AIH (2021a) & No released code, data, or pretrained models. \\
&
\end{tabular}

Table 5: The metrics that were not assessed in this paper and the reasons behind that choice.

human-like.

The Deep AM-FM metric (Zhang et al., 2021b) measures two aspects of dialog quality through the Adequacy Metric (AM) and the Fluency Metric (FM). AM assesses the semantic similarity of system responses and human references by comparing their BERT embeddings. FM compares the similarity of the language model probabilities for both the system response and the human reference, and produces a higher score if the probabilities are similar.

HolisticEval (Pang et al., 2020) evaluates several qualities of dialog: context coherence, language fluency, response diversity, and logical selfconsistency. The GPT-2 language model (Radford et al., 2019) and pretrained Natural Language Inference models are used to measure these qualities.

In addition to measuring the relevance of a response, PredictiveEngage (Ghazarian et al., 2020) incorporates an utterance-level engagement classifier to better assess the overall quality of a response.

FED (Mehri and Eskenazi, 2020a) is an unsupervised evaluation metric that uses DialoGPT (Zhang et al., 2020) to measure 18 fine-grained qualities of dialog. FED calculates the likelihood of manually designed follow-up utterances to measure multiple qualities of dialog without any supervision.

FlowScore (Li et al., 2021b) which is based on the DialoFlow model, models the dynamic information flow in the dialog history to evaluate the quality of a dialog. DialoFlow is a response generation model that is trained with three objectives CFM, SIM and RGM in order to condition the response generation on the context flow in the dialog. FlowScore uses the representations produced by DialoFlow to measure the dialog quality.

FBD (Xiang et al., 2021) computes the distribution-wise difference between the system generated conversations and the human-written conversations to evaluate the performance of a di- alog system. FBD focuses on assessing systemlevel performance and leverages the pretrained RoBERTa model without any additional finetuning.

In addition to automatic metrics specifically designed for dialog evaluation, this paper also evaluates the performance of BERTScore (Zhang et al., 2019), QuestEval (Scialom et al., 2021), and BLEURT (Sellam et al., 2020) which were originally designed for evaluating machine translation, summarization and general natural language generation. BERTScore computes the F1 score by matching token embeddings in the human reference and system response. BLEURT generates synthetic data to pre-train BERT and fine-tune the model to predict a human score with MSE loss. QuestEval, which is based on question generation (QG) and question answering (QA), accounts for factual consistency, relevance, and information selection of the generated response. While these three metrics were not specifically designed for dialog, it is interesting to observe how they perform on dialog data.

\section{A.2 Use of Pretrained Language Models}

Large-scale pretrained language models (Devlin et al., 2019; Radford et al., 2019) have been ubiquitous in NLP models. Embeddings from pretrained language models have been shown to be particularly effective for a variety of NLP tasks. Pretrained models are now a commonly-used strategy in dialog evaluation metrics. However, since different pretrained models use different training data and objectives, the choice of language model might significantly influence the final performance and generalizability of the evaluation metrics. Future work should explore the impact of the different pretrained language models on the performance of the evaluation metric.

BERT (Devlin et al., 2019) is used in many of 
the metrics that this paper assesses. BERT uses the Masked Language Modeling (MLM) and Next Sentence Prediction (NSP) objectives, and is trained on the BookCorpus (Zhu et al., 2015) and English Wikipedia.

RoBERTa, which is employed in USR (Mehri and Eskenazi, 2020b), improves the training techniques in BERT and trains the model on a much larger corpus which includes the CommonCrawl News dataset (Mackenzie et al., 2020) and text extracted from Reddit. Specifically, the full training data size of RoBERTa is 10 times larger than BERT, and empirically RoBERTa has better performance than BERT on common NLP tasks including the GLUE benchmark (Wang et al., 2018).

FED (Mehri and Eskenazi, 2020a) uses DialoGPT (Zhang et al., 2020) which is based on the GPT2 (Radford et al., 2019) architecture and trained with dialog data extracted from Reddit. Due to this, DialoGPT might better model human conversation, particularly open-domain chit-chat.

Deep AM-FM uses Multilingual BERT (Devlin et al., 2019), which is BERT trained on multilingual datasets. The benefits of using multilingual language models to evaluate English data is unclear and is an interesting topic for future work.

T5 (Raffel et al., 2020), which is used by QuestE$\mathrm{val}$, is a Transformer Seq2Seq model pretrained on a massive text corpus. T5 achieved state-of-theart results on many text generation tasks including summarization, question answering, and text classification. The strong performance of T5 on NLG tasks, suggests that it may be valuable in the assessment of response generation models.

\section{A.3 Training Data}

The choice of training data is one of the most important factors in model-based metrics. The domain, quality, and conversation setting of the dataset all play important roles in the relative quality of the resulting metric. For example, a metric trained on Twitter data may also perform well at evaluating dialogs generated for Reddit data, since the two datasets are constructed from online forums. This section introduces the characteristics of the datasets used to train the various model-based metrics.

DailyDialog (Li et al., 2017) is a human-written dialog dataset where the conversation topics are about day-to-day life.

PersonaChat (Zhang et al., 2018) is a dataset that consists of persona-conditioned dialogs where each participant is assigned a persona and the goal is to become familiar with the other individual. In contrast to DailyDialog, dialogs in PersonaChat have a clear objective and are more engaging.

The ConvAI dataset (Dinan et al., 2019) is based on PersonaChat with modifications to preprocessing and additional training examples.

TopicalChat (Gopalakrishnan et al., 2019) consists of knowledge-grounded human-human conversations, wherein two individuals have a conversation grounded on 'interesting facts'. Models trained on TopicalChat are expected to be able to use external knowledge and have realistic knowledgegrounded conversations.

Twitter (Ritter et al., 2011) and Ubuntu Dialogue (Lowe et al., 2015) both result from a crawl of the internet. Ubuntu Dialogue has very technical conversations regarding computer systems, while Twitter covers a broad, general set of topics.

These datasets were generally used to train the metrics through the use of self-supervised objectives.

\section{A.4 Referenced vs Reference-Free}

Word-overlap metrics are ineffective for dialog (Liu et al., 2016b) largely due to the one-to-many nature of dialog (Zhao et al., 2017a). While this could be mitigated by using multiple reference responses, it is infeasible to collect a sufficiently large dataset to thoroughly cover the space of potential responses. Thus, reference-free metrics have been proposed to circumvent the one-to-many problem. Amongst the metrics assessed here, there are several referencefree evaluation metrics: HolisticEval, MAUDE, GRADE, USR, FED, FlowSore, USL-H, QuestEval, DEB, DynaEval, PredictiveEngage and DialogRPT.

In contrast to the referenced metrics, which compare the generated response to the reference response, reference-free metrics model the semantics of the dialog context and the generated response in order to reason about the response within the context of the dialog history.

\section{A.5 Metrics not Assessed in this Paper}

Table 5 lists recently proposed dialog metrics that are not assessed in this paper. There are several reasons a metric was not assessed:

- The metric was not designed specifically for dialog. While most of these metrics were not included, a few metrics that fall into this 
category (e.g., BERTScore, QuestEval, and BLEURT) were assessed here, as a baseline and to represent this category.

- There was no released code, data, or pretrained model for reproducing their results.

- The core idea of the metric is very similar to metrics that were assessed.

- The metric is infeasible to assess in our experimental setting, as it requires additional annotations. This may include requiring human annotations or information about the response generation models.

Some of the unassessed metrics that share ideas that are covered by other metrics assessed in this paper are:

Embedding Methods (Kiros et al., 2015; Liu et al., 2016a; Rodríguez-Cantelar et al., 2021) compute the similarity of system responses with the human reference through embeddings, which is equivalent to the approach used by RUBER and BERTScore.

Language Model Evaluator (Nedelchev et al., 2020) evaluates dialog using the language model likelihood, which is identical to the approaches of USR and HolisticEval.

\section{B Testing Datasets}

\section{B.1 Data Collection}

This section provides an overview of the different quality-annotated dialog datasets. Many of these datasets were collected in different settings. For example, as described in Section A.3, DailyDia$\log$ consists of casual conversations about daily life while TopicalChat consists of knowledge-grounded conversations. These differences influence various aspects of the data, such as the formality and complexity of the sentence structure. However, since it is not easy to quantify the complexity of the sentence structure, the average length and the number of distinct words in the dialog context (Ctx), human reference (Ref) and model hypothesis (Hyp) are used in Table 6 . The length of the context and reference response in DailyDialog is shorter than on TopicalChat, which is likely influenced by the simpler topics in DailyDialog.

GRADE-DailyDialog has a significantly longer dialog history than the other two quality-annotated datasets that use DailyDialog. This is because those two datasets, HolisticEval and PredictiveEngage, only use one utterance of the dialog while
GRADE uses two utterances. On the other hand, responses in PredictiveEngage-DailyDialog use a larger number of distinct words, which is due to the fact that PredictiveEngage-DailyDialog also contains human-written responses in addition to responses generated by NLG systems.

While most of the dialog data was collected by recruiting human annotators via Amazon Mechanical Turks (AMT), the DSTC6 data (Hori and Hori, 2017) uses dialogs from Twitter. Therefore, we can expect the DSTC6 data to be noisier and more realistic. It poses a challenge for both NLG models and evaluation metrics.

In contrast to other quality-annotated datasets in which the dialog contexts are conversations between humans and only the responses are generated by the system, the DSTC9 and FED datasets provide human-system dialogs that were collected in an interactive setting. The DSTC9 data (Gunasekara et al., 2021) was collected on the DialPort platform through direct interaction between real users and open-domain chit-chat systems. The FED data (Mehri and Eskenazi, 2020a) contains both human-human and human-system conversations released by Adiwardana et al. (2020b).

Despite using the same underlying dialog dataset (e.g., DailyDialog), the complexity and quality of the response may differ significantly across the quality-annotated datasets depending on the response generation model that was used. If responses in a quality-annotated dataset are from both simple sequence-to-sequence (Seq2Seq) models (Cho et al., 2014) and state-of-the-art language models such as DialoGPT, this quality-annotated dataset can assess whether the metric can distinguish between high quality and low quality responses.

Distinguishing between responses produced by systems of very different quality is easier, since the low quality responses may not follow grammar rules and make simple mistakes. On the other hand, if responses are only generated by state-of-the-art systems, the task becomes harder because the metric needs to rank responses as to whether they are appropriate in a dialog context.

Responses in USR-TopicalChat, USRPersonaChat, HolisticEval-DailyDialog and the DSTC6 data are generated by a relatively simple model, such as an LSTM language model (LSTM LM), LSTM or Transformer Seq2Seq model, or Memory Network. 


\begin{tabular}{l|ccccl}
\hline Dataset & Num. Samples & Avg. Utts. & Avg. Ctx/Ref/Hyp Words & Distinct Ctx/Ref/Hyp Words & Used NLG models \\
\hline USR-TopicalChat & 300 & 12.2 & $236.3 / 25.3 / 22.4$ & $2379 / 571 / 1018$ & Trans \\
USR-PersonaChat & 240 & 9.3 & $98.4 / 11.7 / 12.1$ & $1320 / 291 / 609$ & Trans Seq2Seq, LSTM LM, Memory Network \\
GRADE-ConvAI2 & 600 & 2.0 & $23.4 / 12.1 / 11.3$ & $1342 / 841 / 1100$ & Trans Seq2Seq, DialoGPT, BERT/Trans Ranker \\
GRADE-DailyDialog & 300 & 2.0 & $24.5 / 12.9 / 10.8$ & $1126 / 674 / 796$ & Trans Seq2Seq, Trans Ranker \\
HolisticEval-DailyDialog & 200 & 1.0 & $14.3 / / / 14.7$ & $847 /-/ 875$ & LSTM Seq2Seq \\
PredictiveEngage-DailyDialog & 600 & 1.0 & $12.0 / / / 13.7$ & $1022 /-/ 2475$ & LSTM Seq2Seq \\
GRADE-ED (2019) & 300 & 2.0 & $28.0 / 17.8 / 15.6$ & $1430 / 1098 / 701$ & Trans Seq2Seq, Trans Ranker \\
DSTC6 (2017) & 40000 & 1.6 & $30.7 / 19.0 / 19.8$ & $7399 / 3410 / 2473$ & Systems based on LSTM Seq2Seq \\
FED (2020a) & 500 & 11.8 & $86.5 / / / 11.8$ & $3628 /-/ 1859$ & Meena, Mitsuku \\
DSTC9 (2021) & 2200 & 27.2 & $231.7 /-/ 10.6$ & $35591 /-/ 4839$ & SOTA systems including Plato and DialoGPT
\end{tabular}

Table 6: Statistics of quality-annotated datasets. Ctx, Ref, and Hyp indicate dialog context, human reference, and model hypothesis, respectively. Trans and ED are the abbreviation of the Transformer and EmpatheticDialogue respectively.

On the other hand, responses in the qualityannotated data labeled by GRADE come from the Transformer Seq2Seq model, DialoGPT, and retrieval model using Transformer or BERT (Transformer/BERT Ranker), which have relatively better empirical performance. Furthermore, FED and DSTC9 data use state-of-the-art dialog systems to generate responses. Specifically, FED data incorporates two systems, Meena (Adiwardana et al., 2020b) and Mitsuku ${ }^{2}$, and the DSTC9 data uses dialog systems including PLATO (Bao et al., 2020). These high quality responses make the data more challenging.

\section{B.2 Quality Annotation}

After generating responses for each dataset, human annotators labeled the quality of each response. While most quality-annotated datasets provide annotations for the overall score of a given response, HolisticEval-DailyDialog only labels the context coherence of a response. ${ }^{3}$. In addition to the overall score, USR and FED data provide annotations for different dialog qualities such as whether the response is coherent or interesting. These finegrained annotations allow for a more comprehensive analysis of metrics.

\section{Experiments}

\section{C.1 Metric Output Similarities}

To analyze the similarities between the metrics, we plot the correlation between metric outputs in Figure 3. As expected, there is a strong correlation between the outputs of BERTSCore, and BLEURT, the rule-based metrics. Interestingly, Deep AMFM also is strongly correlated with these word-

\footnotetext{
${ }^{2}$ https: / / medium. com/pandorabots-blog/ mitsuku-wins-loebner-prize-2018-3e8d98c5f2

${ }^{3}$ HolisticEval also released the data for evaluating response fluency. However since the data does not clearly disambiguate between the dialog context and the corresponding system response, it was not used in this paper
}

overlap metrics. This is surprising since Deep AMFM was specifically designed for evaluating dialog while the others are intended for general-purpose NLG evaluation. This may be because the Deep AM-FM metric compares the generated response to the reference response and, as such, will favor generated responses that have high word-overlap with the reference response.

BERT-RUBER, PONE, GRADE, and USL-H are another group of metrics with similar behavior. Since BERT-RUBER, PONE, and GRADE share a common triplet rank loss and train on DailyDialog, it is not surprising that these three metrics behave similarly. Although USL-H is trained with different objectives, the same behavior might be due to the use of the same training data (DailyDialog) and the same pretrained model (BERT). Moreover, USR has a slightly higher correlation to this group. This is likely because USR aggregates multiple qualities similar to USL-H and models the relevance of responses, similar to the RUBER-based metrics and GRADE.

\section{C.2 Performance on Different Response Generation Models}

This section presents the correlations of the metrics on both generative and retrieval response generation models (see Table 10). Most of the metrics perform similarly on these two types of models. However, USR, GRADE, and FlowScore perform very differently. USR and GRADE are especially good at scoring responses from the retrieval model. USR performs significantly less well at evaluating generative models while GRADE still achieves the highest correlation. In contrast, FlowScore performs well at evaluating generative models but performs poorly on generative models. One reason for this may be that responses from generative models are longer and more complex than the ones from retrieval models. 


\begin{tabular}{|c|c|c|c|c|c|c|c|c|}
\hline & \multicolumn{4}{|c|}{ USR-TopicalChat } & \multicolumn{4}{|c|}{ USR-PersonaChat } \\
\hline & \multicolumn{2}{|c|}{ Turn-Level } & \multicolumn{2}{|c|}{ System-Level } & \multicolumn{2}{|c|}{ Turn-Level } & \multicolumn{2}{|c|}{ System-Level } \\
\hline & $\mathrm{P}$ & $\mathrm{S}$ & $\mathrm{P}$ & $\mathrm{S}$ & $\mathrm{P}$ & $\mathrm{S}$ & $\mathrm{P}$ & $\mathrm{S}$ \\
\hline BLEU-4 & 0.216 & 0.296 & $0.874 *$ & 0.900 & 0.135 & $0.090^{*}$ & $0.841 *$ & $0.800^{*}$ \\
\hline METEOR & 0.336 & 0.391 & 0.943 & 0.900 & 0.253 & 0.271 & $0.907^{*}$ & $0.800^{*}$ \\
\hline ROUGE-L & 0.275 & 0.287 & $0.814^{*}$ & 0.900 & $0.066^{*}$ & $0.038^{*}$ & $0.171 *$ & $0.000^{*}$ \\
\hline ADEM & $-0.060 *$ & $-0.061 *$ & $0.202 *$ & $0.700 *$ & -0.141 & $-0.085^{*}$ & $0.523 *$ & $0.400^{*}$ \\
\hline BERTScore & 0.298 & 0.325 & $0.854 *$ & 0.900 & 0.152 & $0.122^{*}$ & $0.241 *$ & $0.000^{*}$ \\
\hline BLEURT & 0.216 & 0.261 & $0.630 *$ & 0.900 & $0.065^{*}$ & $0.054^{*}$ & $-0.125^{*}$ & $0.000^{*}$ \\
\hline QuestEval & 0.300 & 0.338 & 0.943 & 1.000 & 0.176 & 0.236 & $0.885^{*}$ & 1.000 \\
\hline RUBER & 0.247 & 0.259 & $0.876^{*}$ & 1.000 & 0.131 & 0.190 & 0.997 & 1.000 \\
\hline BERT-RUBER & 0.342 & 0.348 & 0.992 & 0.900 & 0.266 & 0.248 & 0.958 & $0.200^{*}$ \\
\hline PONE & 0.271 & 0.274 & 0.893 & $0.500^{*}$ & 0.373 & 0.375 & 0.979 & $0.800^{*}$ \\
\hline MAUDE & $0.044 *$ & $0.083^{*}$ & $0.317 *$ & $-0.200 *$ & 0.345 & 0.298 & $0.440 *$ & $0.400 *$ \\
\hline DEB & 0.180 & 0.116 & $0.818^{*}$ & $0.400^{*}$ & 0.291 & 0.373 & 0.989 & 1.000 \\
\hline GRADE & 0.200 & 0.217 & $0.553 *$ & $0.100^{*}$ & 0.358 & 0.352 & $0.811^{*}$ & 1.000 \\
\hline DynaEval & $-0.032 *$ & $-0.022 *$ & $-0.248 *$ & $0.100^{*}$ & 0.149 & 0.171 & $0.584 *$ & $0.800 *$ \\
\hline USR & 0.412 & 0.423 & 0.967 & 0.900 & 0.440 & 0.418 & $0.864 *$ & 1.000 \\
\hline USL-H & 0.322 & 0.340 & 0.966 & 0.900 & 0.495 & 0.523 & 0.969 & $0.800 *$ \\
\hline DialogRPT & 0.120 & $0.105^{*}$ & 0.944 & $0.600^{*}$ & $-0.064 *$ & $-0.083^{*}$ & $0.347 *$ & $0.800 *$ \\
\hline Deep AM-FM & 0.285 & 0.268 & 0.969 & $0.700^{*}$ & 0.228 & 0.219 & 0.965 & 1.000 \\
\hline HolisticEval & -0.147 & -0.123 & -0.919 & $-0.200 *$ & $0.087 *$ & $0.113^{*}$ & $0.051 *$ & $0.000^{*}$ \\
\hline PredictiveEngage & 0.222 & 0.310 & $0.870 *$ & 0.900 & $-0.003^{*}$ & $0.033^{*}$ & $0.683^{*}$ & $0.200 *$ \\
\hline FED & -0.124 & -0.135 & $0.730 *$ & $0.100^{*}$ & $-0.028 *$ & $-0.000 *$ & $0.005^{*}$ & $0.400 *$ \\
\hline FlowScore & $0.095^{*}$ & $0.082 *$ & $-0.150 *$ & $0.400 *$ & $0.118^{*}$ & $0.079 *$ & $0.678 *$ & $0.800 *$ \\
\hline FBD & - & - & 0.916 & $0.100^{*}$ & - & - & $0.644 *$ & $0.800^{*}$ \\
\hline
\end{tabular}

Table 7: Results on the USR data. All values are statistically significant to $p<0.05$, unless marked by *.

\begin{tabular}{|c|c|c|c|c|c|c|c|c|c|c|c|c|}
\hline & \multicolumn{4}{|c|}{ GRADE-ConvAI2 } & \multicolumn{4}{|c|}{ GRADE-DailyDialog } & \multicolumn{4}{|c|}{ GRADE-EmpatheticDialogue } \\
\hline & \multicolumn{2}{|c|}{ Turn-Level } & \multicolumn{2}{|c|}{ System-Level } & \multicolumn{2}{|c|}{ Turn-Level } & \multicolumn{2}{|c|}{ System-Level } & \multicolumn{2}{|c|}{ Turn-Level } & \multicolumn{2}{|c|}{ System-Level } \\
\hline & $P$ & $\mathrm{~S}$ & $\mathrm{P}$ & $\mathrm{S}$ & $P$ & $\mathrm{~S}$ & $\mathrm{P}$ & $\mathrm{S}$ & $\mathrm{P}$ & $\mathrm{S}$ & $\mathrm{P}$ & $\mathrm{S}$ \\
\hline BLEU-4 & $0.003 *$ & 0.128 & $0.034 *$ & $0.000^{*}$ & $0.075^{*}$ & 0.184 & $1.000 *$ & 1.000 & $-0.051 *$ & $0.002 *$ & $1.000^{*}$ & 1.000 \\
\hline METEOR & 0.145 & 0.181 & $0.781^{*}$ & $0.600^{*}$ & $0.096^{*}$ & $0.010^{*}$ & $-1.000 *$ & -1.000 & 0.118 & $0.055^{*}$ & $1.000 \%$ & 1.000 \\
\hline ROUGE-L & 0.136 & 0.140 & $0.209^{*}$ & $0.000^{*}$ & 0.154 & 0.147 & $1.000 *$ & 1.000 & $0.029 *$ & $-0.013 *$ & $1.000 *$ & 1.000 \\
\hline ADEM & $-0.060^{*}$ & $-0.057^{*}$ & $-0.368^{*}$ & $-0.200^{*}$ & $0.064 *$ & $0.071^{*}$ & $1.000 *$ & 1.000 & $-0.036 *$ & $-0.028 *$ & $1.000 *$ & 1.000 \\
\hline BERTScore & 0.225 & 0.224 & $0.918^{*}$ & $0.800 *$ & 0.129 & $0.100^{*}$ & $-1.000 *$ & -1.000 & $0.046^{*}$ & $0.033^{*}$ & $1.000^{*}$ & 1.000 \\
\hline BLEURT & 0.125 & 0.120 & $-0.777 *$ & $-0.400^{*}$ & 0.176 & 0.133 & $1.000 *$ & 1.000 & $0.087^{*}$ & $0.051^{*}$ & $1.000^{*}$ & 1.000 \\
\hline QuestEval & 0.279 & 0.319 & $0.283^{*}$ & $0.400^{*}$ & $0.020 *$ & $0.006^{*}$ & $-1.000 *$ & -1.000 & 0.201 & 0.272 & $1.000^{*}$ & 1.000 \\
\hline RUBER & $-0.027 *$ & $-0.042 *$ & $-0.458^{*}$ & $-0.400 *$ & $-0.084 *$ & $-0.094 *$ & $-1.000 *$ & -1.000 & $-0.078^{*}$ & $-0.039 *$ & $1.000^{*}$ & 1.000 \\
\hline BERT-RUBER & 0.309 & 0.314 & $0.885^{*}$ & 1.000 & 0.134 & 0.128 & $-1.000^{*}$ & -1.000 & 0.163 & 0.148 & $1.000^{*}$ & 1.000 \\
\hline PONE & 0.362 & 0.373 & $0.816^{*}$ & $0.800^{*}$ & 0.163 & 0.163 & $-1.000 *$ & -1.000 & 0.177 & 0.161 & $1.000^{*}$ & 1.000 \\
\hline MAUDE & 0.351 & 0.304 & $0.748^{*}$ & $0.800^{*}$ & $-0.036^{*}$ & $-0.073 *$ & $1.000 *$ & 1.000 & $0.007 *$ & $-0.057 *$ & $1.000^{*}$ & 1.000 \\
\hline DEB & 0.426 & 0.504 & 0.995 & 1.000 & 0.337 & 0.363 & $1.000^{*}$ & 1.000 & 0.356 & 0.395 & $1.000^{*}$ & 1.000 \\
\hline GRADE & 0.566 & 0.571 & $0.883^{*}$ & $0.800 *$ & 0.278 & 0.253 & $-1.000 *$ & -1.000 & 0.330 & 0.297 & $1.000 *$ & 1.000 \\
\hline DynaEval & 0.138 & 0.131 & -0.996 & -1.000 & $0.108 *$ & 0.120 & $-1.000 *$ & -1.000 & 0.146 & 0.141 & $-1.000 *$ & -1.000 \\
\hline USR & 0.501 & 0.500 & 0.995 & 1.000 & $0.057 *$ & $0.057 *$ & $-1.000 *$ & -1.000 & 0.264 & 0.255 & $1.000 *$ & 1.000 \\
\hline USL-H & 0.443 & 0.457 & 0.971 & 1.000 & $0.108 *$ & $0.093^{*}$ & $-1.000 *$ & -1.000 & 0.293 & 0.235 & $1.000^{*}$ & 1.000 \\
\hline DialogRPT & 0.137 & 0.158 & $-0.311^{*}$ & $-0.600^{*}$ & $-0.000^{*}$ & $0.037 *$ & $-1.000 *$ & -1.000 & 0.211 & 0.203 & $1.000^{*}$ & 1.000 \\
\hline Deep AM-FM & 0.117 & 0.130 & $0.774 *$ & $0.400 *$ & $0.026^{*}$ & $0.022^{*}$ & $1.000 *$ & 1.000 & $0.083^{*}$ & $0.058^{*}$ & $1.000 *$ & 1.000 \\
\hline HolisticEval & $-0.030 *$ & $-0.010^{*}$ & $-0.297 *$ & $-0.400 *$ & $0.025^{*}$ & $0.020^{*}$ & $1.000 *$ & 1.000 & 0.199 & 0.204 & $-1.000 *$ & -1.000 \\
\hline PredictiveEngage & 0.154 & 0.164 & $0.601 *$ & $0.600 *$ & -0.133 & -0.135 & $-1.000 *$ & -1.000 & $-0.032 *$ & $-0.078 *$ & $1.000 *$ & 1.000 \\
\hline FED & -0.090 & $-0.072 *$ & $-0.254 *$ & $0.000^{*}$ & $0.080^{*}$ & $0.064 *$ & $1.000 *$ & 1.000 & $-0.014 *$ & $-0.044 *$ & $1.000^{*}$ & 1.000 \\
\hline FlowScore & - & - & - & - & - & - & - & - & - & - & - & - \\
\hline FBD & - & - & $-0.235^{*}$ & $-0.400 *$ & - & 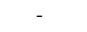 & $-1.000^{*}$ & -1.000 & . & - & $-1.000^{*}$ & -1.000 \\
\hline
\end{tabular}

Table 8: Results on the GRADE data. All values are statistically significant to $p<0.05$, unless marked by *. 


\begin{tabular}{lcccc}
\hline & \multicolumn{4}{c}{ DSTC6 } \\
& \multicolumn{2}{c}{ Turn-Level } & System-Level \\
& $\mathrm{P}$ & $\mathrm{S}$ & $\mathrm{P}$ & $\mathrm{S}$ \\
\hline BLEU-4 & 0.131 & 0.298 & $-0.064^{*}$ & $0.050^{*}$ \\
METEOR & 0.307 & 0.323 & 0.633 & $0.084^{*}$ \\
ROUGE-L & 0.332 & 0.326 & 0.487 & $0.215^{*}$ \\
ADEM & 0.151 & 0.118 & $0.042^{*}$ & $0.347^{*}$ \\
BERTScore & $\mathbf{0 . 3 6 9}$ & $\mathbf{0 . 3 3 7}$ & 0.671 & $0.265^{*}$ \\
BLEURT & 0.326 & 0.294 & $0.213^{*}$ & $0.426^{*}$ \\
QuestEval & 0.188 & 0.242 & $-0.215^{*}$ & $0.206^{*}$ \\
RUBER & 0.114 & 0.092 & $-0.074^{*}$ & $0.104^{*}$ \\
BERT-RUBER & 0.204 & 0.217 & $\mathbf{0 . 8 2 5}$ & $0.093^{*}$ \\
PONE & 0.208 & 0.200 & 0.608 & $0.235^{*}$ \\
MAUDE & 0.195 & 0.128 & 0.739 & $0.217^{*}$ \\
DEB & 0.211 & 0.214 & $-0.261^{*}$ & 0.492 \\
GRADE & 0.119 & 0.122 & 0.784 & 0.611 \\
DynaEval & 0.286 & 0.246 & $0.342^{*}$ & $-0.050^{*}$ \\
USR & 0.184 & 0.166 & $0.432^{*}$ & $0.147^{*}$ \\
USL-H & 0.217 & 0.179 & 0.811 & $0.298^{*}$ \\
DialogRPT & 0.170 & 0.155 & 0.567 & $0.334^{*}$ \\
Deep AM-FM & 0.326 & 0.295 & 0.817 & $\mathbf{0 . 6 7 4}$ \\
HolisticEval & $0.001 *$ & $-0.004^{*}$ & 0.010 & -0.002 \\
PredictiveEngage & 0.043 & $0.004^{*}$ & $-0.094^{*}$ & $-0.409^{*}$ \\
FED & -0.106 & -0.083 & $0.221^{*}$ & $0.322^{*}$ \\
FlowScore & 0.064 & 0.095 & $0.352^{*}$ & $0.362^{*}$ \\
FBD & - & - & -0.481 & $-0.234^{*}$
\end{tabular}

Table 9: Results on the DSTC6 data. All values are statistically significant to $p<0.05$, unless marked by *.



(a) Pearson

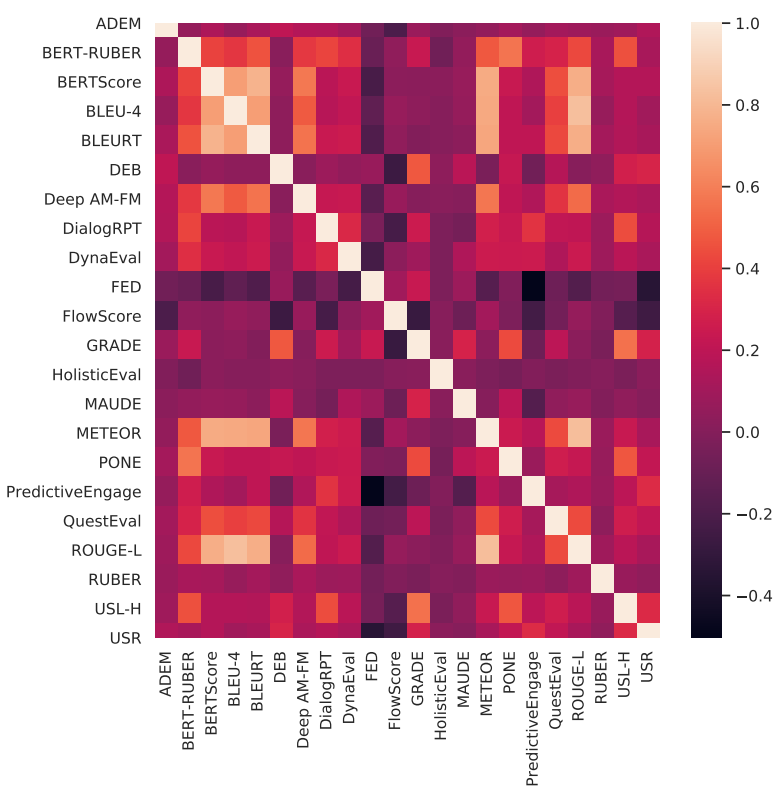

(b) Spearman

Figure 3: Pearson and Spearman correlation between different system outputs. We use datasets with human references to compute scores. 


\begin{tabular}{lcccc}
\hline & \multicolumn{2}{c}{ Generative } & \multicolumn{2}{c}{ Retrieval } \\
& $\mathrm{P}$ & $\mathrm{S}$ & $\mathrm{P}$ & $\mathrm{S}$ \\
\hline BLEU-4 & 0.100 & 0.128 & $0.023^{*}$ & 0.236 \\
METEOR & 0.130 & 0.086 & 0.129 & 0.185 \\
ROUGE-L & 0.134 & 0.108 & 0.212 & 0.238 \\
ADEM & -0.117 & -0.118 & $-0.045^{*}$ & $-0.070^{*}$ \\
BERTScore & 0.182 & 0.185 & 0.175 & 0.186 \\
BLEURT & 0.153 & 0.147 & 0.108 & 0.093 \\
QuestEval & 0.115 & 0.112 & 0.105 & 0.190 \\
RUBER & $0.022^{*}$ & $0.031^{*}$ & -0.092 & -0.109 \\
BERT-RUBER & 0.158 & 0.159 & 0.206 & 0.208 \\
PONE & 0.227 & 0.236 & 0.191 & 0.192 \\
MAUDE & 0.126 & 0.161 & $0.072^{*}$ & $0.031^{*}$ \\
DEB & 0.281 & 0.313 & 0.307 & 0.420 \\
GRADE & $\mathbf{0 . 3 0 0}$ & $\mathbf{0 . 3 1 4}$ & $\mathbf{0 . 4 3 5}$ & $\mathbf{0 . 4 2 8}$ \\
DynaEval & 0.110 & 0.113 & 0.143 & 0.157 \\
USR & 0.130 & 0.149 & 0.402 & 0.415 \\
USL-H & 0.126 & 0.161 & 0.307 & 0.330 \\
DialogRPT & -0.082 & -0.067 & $-0.009^{*}$ & $0.001^{*}$ \\
Deep AM-FM & 0.068 & 0.068 & 0.086 & 0.085 \\
HolisticEval & $-0.037 *$ & $-0.003 *$ & $0.070^{*}$ & $0.066^{*}$ \\
PredictiveEngage & $-0.063^{*}$ & $-0.060^{*}$ & $0.010^{*}$ & $0.028^{*}$ \\
FED & -0.072 & -0.095 & -0.169 & -0.184 \\
FlowScore & 0.236 & 0.215 & $-0.059^{*}$ & $-0.053^{*}$ \\
FBD & - & - & - & -
\end{tabular}

Table 10: Results on evaluating different response generation models. All values are statistically significant to $p<0.05$, unless marked by $*$.

\begin{tabular}{|c|c|c|c|c|c|c|c|c|c|c|c|c|}
\hline & \multicolumn{2}{|c|}{ USR-TopicalChat } & \multicolumn{2}{|c|}{ USR-PersonaChat } & \multicolumn{2}{|c|}{ GRADE-ConvAI2 } & \multicolumn{2}{|c|}{ GRADE-DailyDialog } & \multicolumn{2}{|c|}{ GRADE-EmpatheticDialogue } & \multicolumn{2}{|c|}{ DSTC6 } \\
\hline & $\mathrm{P}$ & $\mathrm{s}$ & $\mathrm{P}$ & $\mathrm{S}$ & $\mathrm{P}$ & $\mathrm{s}$ & $\mathrm{P}$ & $\mathrm{s}$ & $\mathrm{P}$ & $\mathrm{s}$ & $\mathrm{P}$ & $\mathrm{s}$ \\
\hline PredictiveEngage & 0.222 & 0.310 & $-0.003 *$ & $0.033 *$ & 0.154 & 0.164 & -0.133 & -0.135 & $-0.032 *$ & $-0.078^{*}$ & 0.043 & $0.004 *$ \\
\hline + RUBER & 0.283 & 0.327 & $0.057 *$ & $0.096 *$ & 0.105 & 0.102 & -0.149 & -0.156 & $-0.065 *$ & $-0.055^{*}$ & 0.103 & 0.055 \\
\hline+ PONE & 0.308 & 0.350 & 0.218 & 0.209 & 0.333 & 0.339 & $-0.034 *$ & $-0.035^{*}$ & $0.077 *$ & $0.069^{*}$ & 0.156 & 0.122 \\
\hline USR & 0.412 & 0.423 & 0.440 & 0.418 & 0.501 & 0.500 & $0.057 *$ & $0.057 *$ & 0.264 & 0.255 & 0.184 & 0.166 \\
\hline + GRADE & 0.424 & 0.432 & 0.456 & 0.430 & 0.523 & 0.528 & $0.081 *$ & $0.078^{*}$ & 0.281 & 0.267 & 0.191 & 0.173 \\
\hline+ USL-H & 0.429 & 0.440 & 0.468 & 0.451 & 0.517 & 0.522 & $0.062 *$ & $0.062 *$ & 0.278 & 0.265 & 0.197 & 0.178 \\
\hline$+\mathrm{DEB}$ & 0.359 & 0.362 & 0.453 & 0.473 & 0.552 & 0.551 & 0.222 & 0.190 & 0.391 & 0.385 & 0.248 & 0.234 \\
\hline + GRADE + PONE & 0.435 & 0.447 & 0.473 & 0.452 & 0.535 & 0.539 & 0.089 & 0.086 & 0.289 & 0.270 & 0.206 & 0.187 \\
\hline + GRADE + PONE + PE & 0.447 & 0.468 & 0.468 & 0.449 & 0.534 & 0.538 & $0.072 *$ & $0.070 *$ & 0.275 & 0.261 & 0.202 & 0.180 \\
\hline + GRADE + USL-H + DEB & 0.377 & 0.382 & 0.476 & 0.495 & 0.571 & 0.579 & 0.229 & 0.198 & 0.399 & 0.387 & 0.254 & 0.238 \\
\hline GRADE & 0.200 & 0.217 & 0.358 & 0.352 & 0.566 & 0.571 & 0.278 & 0.253 & 0.330 & 0.297 & 0.119 & 0.122 \\
\hline+ PONE & 0.282 & 0.297 & 0.435 & 0.436 & 0.547 & 0.556 & 0.275 & 0.279 & 0.306 & 0.279 & 0.191 & 0.179 \\
\hline$+\mathrm{PONE}+\mathrm{PE}$ & 0.335 & 0.348 & 0.373 & 0.366 & 0.515 & 0.530 & 0.118 & 0.117 & 0.219 & 0.186 & 0.181 & 0.155 \\
\hline+ USL-H & 0.302 & 0.304 & 0.493 & 0.480 & 0.551 & 0.556 & 0.229 & 0.237 & 0.342 & 0.307 & 0.185 & 0.169 \\
\hline+ DEB & 0.201 & 0.217 & 0.319 & 0.403 & 0.463 & 0.549 & 0.345 & 0.325 & 0.368 & 0.391 & 0.214 & 0.200 \\
\hline + DEB + USL-H & 0.219 & 0.270 & 0.347 & 0.465 & 0.484 & 0.547 & 0.339 & 0.311 & 0.377 & 0.395 & 0.225 & 0.215 \\
\hline USL-H & 0.322 & 0.340 & 0.495 & 0.523 & 0.443 & 0.457 & $0.108 *$ & $0.093^{*}$ & 0.293 & 0.235 & 0.217 & 0.179 \\
\hline$+\mathrm{DEB}$ & 0.201 & 0.269 & 0.322 & 0.482 & 0.452 & 0.501 & 0.333 & 0.258 & 0.366 & 0.390 & 0.224 & 0.223 \\
\hline All-Metrics & 0.459 & 0.473 & 0.522 & 0.534 & 0.566 & 0.561 & 0.163 & 0.149 & 0.366 & 0.349 & 0.288 & 0.268 \\
\hline
\end{tabular}

Table 11: Results of different combinations of metrics. All values are statistically significant to $p<0.05$, unless marked by *. PE is the abbreviation of PredictiveEngage. The last row (All-Metrics), is the average of all the metrics. 


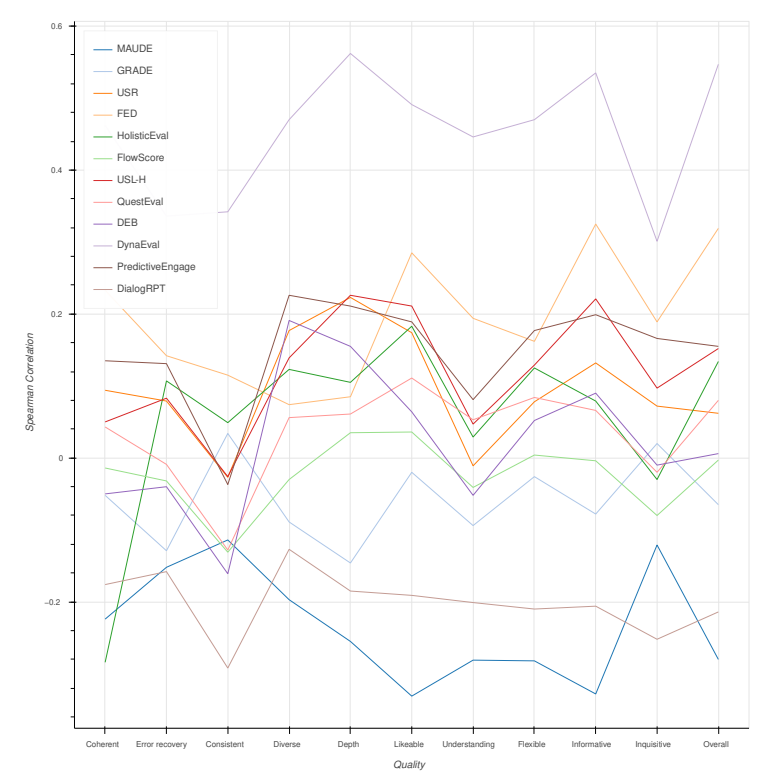

Figure 4: Spearman correlation to dialog-level annotation qualities on the FED data.

\section{C.3 Combining Metrics}

Many metrics rely on an ensemble of different models. Inspired by this, this section explores the possibility that a combination of metrics may be successful (Table 11). Since it is not feasible to exhaustively explore all possible combinations of the metrics, some combinations of the best-performing metrics were explored here. The metrics are combined through simple averaging. Future work should explore more sophisticated mechanisms for combining metrics.

First, PredictiveEngage, RUBER and PONE were combined, as proposed in (Ghazarian et al., 2020). Indeed, the resulting combined metric shows significant improvement, especially on the USR-TopicalChat and USR-Personachat data, but correlation decreases on other datasets.

Next, combinations of USR, GRADE, USL-H and DEB (the best performing metrics on the referenced quality-annotated datasets) were explored. The combination of USR with GRADE, USL-H, PONE and PredictiveEngage performs better on the USR data as well as on GRADE-ConvAI2. Given the strong performance of GRADE on the GRADE data, combining it with the other metrics negatively impacts its performance. However, combining GRADE and DEB does result in an improvement. Interestingly, combining DEB with USR and USL-H also negatively affects the results. In Figure 3, we observe that the outputs of GRADE and DEB have high correlation to each other, which is an indication of the similar behavior of the two metrics. The output similarities of metrics may be a good indicator for how to best combine metrics.

The last row of Table 11, shows the result of taking the average of the scores of all of the metrics that were assessed in this paper. While the idea of an All metric is simple, and metrics are combined through simple averaging, the results are surprisingly good across the different referenced quality-annotated datasets. This result highlights the potential of combining various evaluation metrics in some smart manner. 